\title{
On the number of paths of length 5 in a graph
}

\author{
Nazanin Movarraei*, S. A. Boxwala \\ Department of Mathematics, University of Pune, Pune 411007 , India \\ *Corresponding author E-mail: nazanin.movarraei@gmail.com
}

Copyright (C)2014 Nazanin Movarraei, S. A. Boxwala. This is an open access article distributed under the Creative Commons Attribution License, which permits unrestricted use, distribution, and reproduction in any medium, provided the original work is properly cited.

\begin{abstract}
In this paper, we obtain an explicit formula for the total number of paths of length 5 in a simple graph G. We also determine some formulae for the number of paths of length 5 each of which starts from a specific vertex $v_{i}$ and for the number of $v_{i}-v_{j}$ paths of length 5 in a simple graph $\mathrm{G}$, in terms of the adjacency matrix and with the help of combinatorics.
\end{abstract}

Keywords: Adjacency Matrix; Cycle; Graph Theory; Path; Subgraph, Walk.

\section{Introduction}

In a simple graph $\mathrm{G}$, a walk is a sequence of vertices and edges of the form $v_{0}, e_{1}, v_{1}, \ldots, e_{k}, v_{k}$ such that the edge $e_{i}$ has ends $v_{i-1}$ and $v_{i}$. A walk is called closed if $v_{0}=v_{k}$. If the vertices of a walk are distinct then the walk is called a path. A cycle is a non-trivial closed walk in which all the vertices are distinct except the end vertices.

It is known that if a graph $\mathrm{G}$ has adjacency matrix $\mathrm{A}=\left[a_{i j}\right]$, then for $\mathrm{k}=0,1, \ldots$, the ij-entry of $\mathrm{A}^{k}$ is the number of $v_{i}-v_{j}$ walks of length $\mathrm{k}$ in $\mathrm{G}$. It is also known that $\operatorname{tr}\left(\mathrm{A}^{n}\right)$ is the sum of the diagonal entries of $\mathrm{A}^{n}$ and $d_{i}$ is the degree of the vertex $v_{i}$.

In 1971, Frank Harary and Bennet Manvel [2], gave formulae for the number of cycles of lengths 3 and 4 in simple graphs as given by the following theorems:

Theorem 1.1 [2] If $G$ is a simple graph with adjacency matrix $A$, then the number of 3 -cycles in $G$ is $\frac{1}{6}$ tr $\left(A^{3}\right)$.

(It is known that $\operatorname{tr}\left(A^{3}\right)=\sum_{i=1}^{n} a_{i i}^{(3)}=\sum_{j \neq i} a_{i j}^{(2)} a_{i j}$ ).

Theorem 1.2 [2] If $G$ is a simple graph with adjacency matrix $A$, then the number of $4-$ cycles in $G$ is $\frac{1}{8}\left[\operatorname{tr}\left(A^{4}\right)-2 q-2 \sum_{j \neq i} a_{i j}^{(2)}\right]$, where $q$ is the number of edges in $G$.

(It is obvious that the above formula is also equal to $\frac{1}{8}\left[\operatorname{tr} A^{4}-\operatorname{tr} A^{2}-2 \sum_{j \neq i} a_{i j}^{(2)}\right]$ )

They also gave a formula for the number of 5-cycles in a simple graph. Their proofs are based on the following fact: The number of $\mathrm{n}$-cycles $(\mathrm{n}=3,4,5)$ in a graph $\mathrm{G}$ is equal to $\frac{1}{2 n}\left(\operatorname{tr}\left(\mathrm{A}^{n}\right)-x\right)$ where $x$ is the number of closed walks of length $\mathrm{n}$, which are not n-cycles. 
In 1986, Tomescu [4], gave some formulae for the number of paths of length $\mathrm{s}$, having $\mathrm{k}$ edges in common with a fixed s-path of a complete graph. In 1994, Bax [5], gave an algorithm to count number of all paths and $v_{i}-v_{j}$ paths in a graph. His algorithm cannot count the number of paths of a specific size.

In 1996, Eric Bax and Joel Franklin [7], gave an algorithm to count paths and cycles of a given length in a directed graph. In $[6,8,9,10,12,13,15]$, we have also some bounds to estimate the total time complexity for finding or counting paths and cycles in a graph.

In the previous works there is no formula to count the exact number of paths of a specific size in a graph.

In our recent work [1], we obtained some formulae and propositions to find the exact number of paths of lengths 3 and 4 , in a simple graph $\mathrm{G}$, given below:

Proposition 1.3 [1] In a simple graph $G$ with $n$ vertices and the adjacency matrix $A=\left[a_{i j}\right]$, the number of paths of length $n$ is $\sum_{j \neq i} a_{i j}^{(n)}-x$, where $x$ is the number of non-closed walks of length $n$ in $G$, which are not paths.

Proposition 1.4 [1] In a simple graph $G$ with $n$ vertices and the adjacency matrix $A=\left[a_{i j}\right]$, the number of paths of length $n$, each of which begins with a specific vertex $v_{i}$ is $\sum_{j=1, j \neq i}^{n} a_{i j}^{(n)}-x$, where $x$ is the number of non-closed walks of length $n$ in $G$, starting from the vertex $v_{i}$, which are not paths.

Proposition 1.5 [1] In a simple graph $G$ with $n$ vertices and the adjacency matrix $A=\left[a_{i j}\right]$, the number of $v_{i}-v_{j}$ $(j \neq i)$ paths of length $n$ is $a_{i j}^{(n)}-x$, where $x$ is the number of non-closed $v_{i}-v_{j}$ walks of length $n$ in $G$, which are not paths.

Theorem 1.6 [1] Let $G$ be a simple graph with $n$ vertices and the adjacency matrix $A=\left[a_{i j}\right]$. The number of paths of length 3 in $G$ is $\sum_{j \neq i} a_{i j}^{(2)}\left(d_{j}-a_{i j}-1\right)$.

Theorem 1.7 [1] Let $G$ be a simple graph with $n$ vertices and the adjacency matrix $A=\left[a_{i j}\right]$. The number of paths of length 4 in $G$ is $\sum_{j \neq i}\left[a_{i j}^{(4)}-2 a_{i j}^{(2)}\left(d_{j}-a_{i j}\right)\right]-\sum_{i=1}^{n}\left[\left(2 d_{i}-1\right) a_{i i}^{(3)}+6\left(\begin{array}{c}d_{i} \\ 3\end{array}\right)\right]$.

Theorem 1.8 [1] Let $G$ be a simple graph with $n$ vertices and the adjacency matrix $A=\left[a_{i j}\right]$. The number of paths of length 3 in $G$, each of which starts from a specific vertex $v_{i}$ is $\sum_{j=1, j \neq i}^{n} a_{i j}^{(2)}\left(d_{j}-a_{i j}-1\right)$.

Theorem 1.9 [1] Let $G$ be a simple graph with $n$ vertices and the adjacency matrix $A=\left[a_{i j}\right]$. The number of paths of length 4 in $G$, each of which starts from a specific vertex $v_{i}$ is $\sum_{j=1, j \neq i}^{n}\left[a_{i j}^{(4)}-\left(d_{i}+d_{j}-3 a_{i j}\right) a_{i j}^{(2)}-\left(a_{i i}^{(3)}+a_{j j}^{(3)}+\right.\right.$ $\left.\left.2\left(\begin{array}{c}d_{j}-1 \\ 2\end{array}\right)\right) a_{i j}\right]$

Theorem 1.10 [1] Let $G$ be a simple graph with $n$ vertices and the adjacency matrix $A=\left[a_{i j}\right]$. The number of $v_{i}-v_{j}(j \neq i)$ paths of length 3 in $G$ is $\sum_{k=1, k \neq i, j}^{n}\left(a_{i k}^{(2)}-a_{i j}\right) a_{j k}$.

In this paper we give some formulae to count the exact number of paths of length 5 in a simple graph $G$, in terms of the adjacency matrix of $\mathrm{G}$ and with the help of combinatorics.

\section{Number of paths of length 5}

In this section, we give formulae to count the number of paths of length 5 in a simple graph G. We first give a result below which is useful to prove our other theorems. 
Theorem 2.1 If $G$ is a simple graph with $n$ vertices and the adjacency matrix $A=\left[a_{i j}\right]$, then the number of 4 -cycles each of which contains a specific vertex $v_{i}$ of $G$ is $\frac{1}{2}\left[a_{i i}^{(4)}-a_{i i}^{(2)}-2\left(\begin{array}{c}d_{i} \\ 2\end{array}\right)-\sum_{j=1, j \neq i}^{n} a_{i j}^{(2)}\right]$.

Proof: The number of 4 -cycles each of which contains a specific vertex $v_{i}$ of the graph $\mathrm{G}$ is equal to $\frac{1}{2}\left(a_{i i}^{(4)}-x\right)$, where $x$ is the number of closed walks of length 4 from the vertex $v_{i}$ to $v_{i}$ that are not $4-$ cycles. To find $x$, we have 3 cases as considered below; the cases are based on the configurations-(subgraphs) that generate $v_{i}-v_{i}$ walks of length 4 that are not cycles. In each case, $\mathrm{N}$ denote the number of walks of length 4 from $v_{i}$ to $v_{i}$ that are not cycles in the corresponding subgraph, $\mathrm{M}$ denote the number of subgraphs of $\mathrm{G}$ of the same configuration and $\mathrm{F}$ denote the total number of $v_{i}-v_{i}$ walks of length 4 that are not cycles in all possible subgraphs of G of the same configuration. It is clear that $\mathrm{F}$ is equal to $\mathrm{N} \times \mathrm{M}$. To find $\mathrm{N}$ in each case, we have to include in any walk, all the edges and the vertices of the corresponding subgraphs at least once.

Case 1: For the configuration of Fig $1, \mathrm{~N}=1, \mathrm{M}=a_{i i}^{(2)}, \mathrm{F}=a_{i i}^{(2)}$.

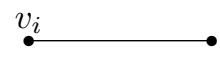

Fig 1

Case 2: For the configuration of Fig $2, \mathrm{~N}=2, \mathrm{M}=\left(\begin{array}{c}d_{i} \\ 2\end{array}\right), \mathrm{F}=2\left(\begin{array}{c}d_{i} \\ 2\end{array}\right)$.

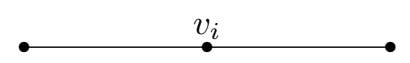

Fig 2

Case 3: For the configuration of Fig 3, $\mathrm{N}=1, \mathrm{M}=\sum_{j=1, j \neq i}^{n} a_{i j}^{(2)}, \mathrm{F}=\sum_{j=1, j \neq i}^{n} a_{i j}^{(2)}$.

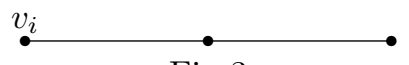

Fig 3

Consequently, $x=a_{i i}^{(2)}+2\left(\begin{array}{c}d_{i} \\ 2\end{array}\right)+\sum_{j=1, j \neq i}^{n} a_{i j}^{(2)}$ and we get the required result.

Example 2.2 In the graph of Fig 4, we have $a_{11}^{(4)}=21, a_{11}^{(2)}=3,2\left(\begin{array}{c}d_{1} \\ 2\end{array}\right)=6, \sum_{j=2}^{4} a_{1 j}^{(2)}=6$. So, by Theorem 2.1, the number of 4-cycles each of which contains the vertex $v_{1}$ in the graph of Fig 4 is 3 .

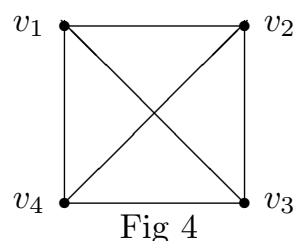

Theorem 2.3 Let $G$ be a simple graph with $n$ vertices and the adjacency matrix $A=\left[a_{i j}\right]$. The number of paths of length 5 in $G$ is $\sum_{j \neq i} a_{i j}^{(5)}-2 \sum_{j \neq i} a_{i j}^{(4)}+2 \sum_{i=1}^{n} a_{i i}^{(3)}\left(d_{i}-2\right)+4 \sum_{j \neq i} a_{i j}^{(2)}-2 \sum_{j \neq i} a_{i j}^{(2)}\left(d_{j}-a_{i j}-1\right)-4 \sum_{j \neq i} a_{i j}^{(2)}\left(\begin{array}{c}d_{i}-a_{i j}-1 \\ 2\end{array}\right)$ $+6 \sum_{j \neq i} a_{i j}\left(\begin{array}{c}a_{i j}^{(2)} \\ 2\end{array}\right)-2 \sum_{j \neq i} a_{i i}^{(3)} a_{i j}^{(2)}-2 \sum_{i=1}^{n} a_{i i}^{(3)}\left(\begin{array}{c}d_{i}-2 \\ 2\end{array}\right)-2 \sum_{i=1}^{n}\left(a_{i i}^{(4)}-a_{i i}^{(2)}-2\left(\begin{array}{c}d_{i} \\ 2\end{array}\right)-\sum_{j=1, j \neq i}^{n} a_{i j}^{(2)}\right)\left(d_{i}-2\right)-\sum_{j \neq i} a_{i j}-$ $3 \operatorname{tr} A^{4}+6 \operatorname{tr} A^{3}+3 \operatorname{tr} A^{2}$.

Proof: By Proposition 1.3, the number of paths of length 5 in a graph $\mathrm{G}$ is equal to $\sum_{j \neq i} a_{i j}^{(5)}-x$, where $x$ is the number of non-closed walks of length 5 , that are not paths. To find $x$, we have 13 cases as considered below; 
the cases are based on the configurations-(subgraphs) that generate all non-closed walks of length 5 , that are not paths. In each case, $\mathrm{N}$ denote the number of non-closed walks of length 5 , that are not paths in the corresponding subgraph, $\mathrm{M}$ denote the number of subgraphs of $\mathrm{G}$ of the same configuration and $\mathrm{F}$ denote the total number of non-closed walks of length 5 , that are not paths in all possible subgraphs of $\mathrm{G}$ of the same configuration. However, in the cases with more than one figure (cases 7, 12), N, M and F are based on the first graph of the respective figures and $\mathrm{P}_{1}, \mathrm{P}_{2}, \ldots$ denote the number of subgraphs of $\mathrm{G}$ which do not have the same configuration as the first graph but are counted in $\mathrm{M}$. It is clear that $\mathrm{F}$ is equal to $\mathrm{N} \times\left(\mathrm{M}-\mathrm{P}_{1}-\mathrm{P}_{2}-\ldots\right)$. To find $\mathrm{N}$ in each case, we have to include in any walk, all the edges and the vertices of the corresponding subgraphs at least once.

Case 1: For the configuration of Fig $5, \mathrm{~N}=2, \mathrm{M}=\frac{1}{2} \sum_{j \neq i} a_{i j}$ and $\mathrm{F}=\sum_{j \neq i} a_{i j}$.

Fig 5

Case 2: For the configuration of Fig $6, \mathrm{~N}=12, \mathrm{M}=\frac{1}{2} \sum_{j \neq i} a_{i j}^{(2)}$ and $\mathrm{F}=6 \sum_{j \neq i} a_{i j}^{(2)}$.

Fig 6

Case 3: For the configuration of Fig $7, \mathrm{~N}=12, \mathrm{M}=\frac{1}{2} \sum_{j \neq i} a_{i j}^{(2)}\left(d_{j}-a_{i j}-1\right)$ and $\mathrm{F}=6 \sum_{j \neq i} a_{i j}^{(2)}\left(d_{j}-a_{i j}-1\right)$. (See Theorem 1.6)

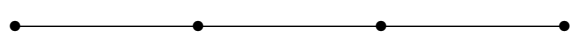

Fig 7

Case 4: For the configuration of Fig $8, \mathrm{~N}=4, \mathrm{M}=\frac{1}{2}\left[\sum_{j \neq i}\left[a_{i j}^{(4)}-2 a_{i j}^{(2)}\left(d_{j}-a_{i j}\right)\right]-\sum_{i=1}^{n}\left[\left(2 d_{i}-1\right) a_{i i}^{(3)}+6\left(\begin{array}{c}d_{i} \\ 3\end{array}\right)\right]\right]$ and $\mathrm{F}=2 \sum_{j \neq i}\left[a_{i j}^{(4)}-2 a_{i j}^{(2)}\left(d_{j}-a_{i j}\right)\right]-2 \sum_{i=1}^{n}\left[\left(2 d_{i}-1\right) a_{i i}^{(3)}+6\left(\begin{array}{c}d_{i} \\ 3\end{array}\right)\right]$. (See Theorem 1.7)

Fig 8

Case 5: For the configuration of Fig $9, \mathrm{~N}=24, \mathrm{M}=\frac{1}{6} \operatorname{tr} A^{3}$ and $\mathrm{F}=4 \operatorname{tr} A^{3}$. (See Theorem 1.1)

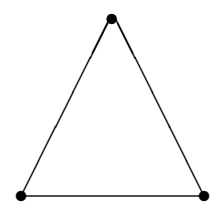

Fig 9

Case 6: For the configuration of Fig 10, $\mathrm{N}=12, \mathrm{M}=\frac{1}{2} \sum_{i=1}^{n} a_{i i}^{(3)}\left(d_{i}-2\right)$ and $\mathrm{F}=6 \sum_{i=1}^{n} a_{i i}^{(3)}\left(d_{i}-2\right)$.

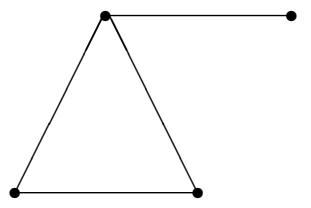

Fig 10

Case 7: For the configuration of Fig $11(\mathrm{a}), \mathrm{N}=4, \mathrm{M}=\frac{1}{2} \sum_{j \neq i} a_{i i}^{(3)} a_{i j}^{(2)}$. Let $\mathrm{P}_{1}$ denote the number of all subgraphs of $\mathrm{G}$ that have the same configuration as the graph of Fig $11(\mathrm{~b})$ and are counted in M. Thus $\mathrm{P}_{1}=6 \times \frac{1}{6} \times \operatorname{tr} A^{3}$, where 
$\frac{1}{6} \times \operatorname{tr} A^{3}$ is the number of subgraphs of $\mathrm{G}$ that have the same configuration as the graph of Fig 11(b) (See Theorem 1.1) and 6 is the number of times that this subgraph is counted in M. Let $\mathrm{P}_{2}$ denote the number of all subgraphs of $\mathrm{G}$ that have the same configuration as the graph of Fig $11(\mathrm{c})$ and are counted in M. Thus $\mathrm{P}_{2}=2 \times \frac{1}{2} \times \sum_{i=1}^{n} a_{i i}^{(3)}\left(d_{i}-2\right)$, where $\frac{1}{2} \times \sum_{i=1}^{n} a_{i i}^{(3)}\left(d_{i}-2\right)$ is the number of subgraphs of $\mathrm{G}$ that have the same configuration as the graph of Fig 11(c) and 2 is the number of times that this subgraph is counted in $\mathrm{M}$. Let $\mathrm{P}_{3}$ denote the number of all subgraphs of $\mathrm{G}$ that have the same configuration as the graph of Fig $11(\mathrm{~d})$ and are counted in M. Thus $\mathrm{P}_{3}=4 \times \frac{1}{2} \times \sum_{j \neq i}\left(\begin{array}{c}a_{i j}^{(2)} \\ 2\end{array}\right) a_{i j}$, where $\frac{1}{2} \times \sum_{j \neq i}\left(\begin{array}{c}a_{i j}^{(2)} \\ 2\end{array}\right) a_{i j}$ is the number of subgraphs of $\mathrm{G}$ that have the same configuration as the graph of Fig $11(\mathrm{~d})$ and 4 is the number of times that this subgraph is counted in M.

Consequently, $\mathrm{F}=2 \sum_{j \neq i} a_{i i}^{(3)} a_{i j}^{(2)}-4 \operatorname{tr} A^{3}-4 \sum_{i=1}^{n} a_{i i}^{(3)}\left(d_{i}-2\right)-8 \sum_{j \neq i}\left(\begin{array}{c}a_{i j}^{(2)} \\ 2\end{array}\right) a_{i j}$.

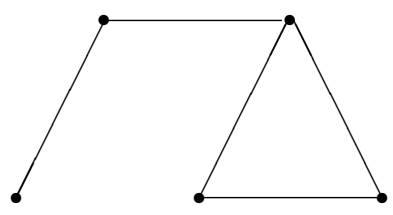

$(a)$

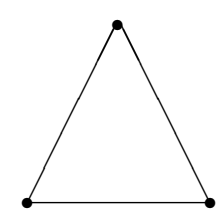

(b)

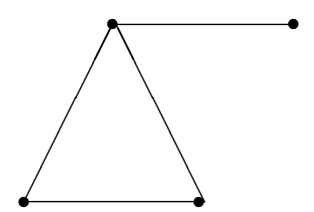

(c)

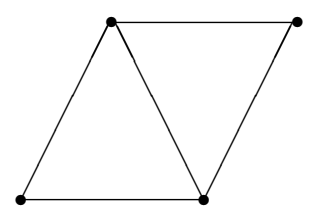

(d)

Case 8: For the configuration of Fig $12, \mathrm{~N}=4, \mathrm{M}=\frac{1}{2} \sum_{i=1}^{n} a_{i i}^{(3)}\left(\begin{array}{c}d_{i}-2 \\ 2\end{array}\right)$ and $\mathrm{F}=2 \sum_{i=1}^{n} a_{i i}^{(3)}\left(\begin{array}{c}d_{i}-2 \\ 2\end{array}\right)$.

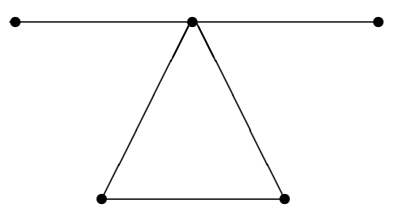

Fig 12

Case 9: For the configuration of Fig 13, $\mathrm{N}=12, \mathrm{M}=\sum_{i=1}^{n}\left(\begin{array}{c}d_{i} \\ 3\end{array}\right)$ and $\mathrm{F}=12 \sum_{i=1}^{n}\left(\begin{array}{c}d_{i} \\ 3\end{array}\right)$.

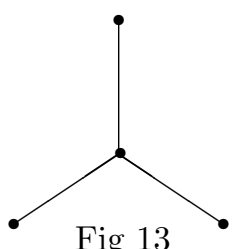

Case 10: For the configuration of Fig 14, $\mathrm{N}=12, \mathrm{M}=\frac{1}{2} \sum_{j \neq i}\left(\begin{array}{c}a_{i j}^{(2)} \\ 2\end{array}\right) a_{i j}$ and $\mathrm{F}=6 \sum_{j \neq i}\left(\begin{array}{c}a_{i j}^{(2)} \\ 2\end{array}\right) a_{i j}$.

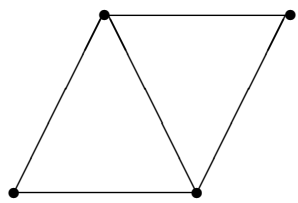

Fig 14 
Case 11: For the configuration of Fig 15, $\mathrm{N}=24, \mathrm{M}=\frac{1}{8}\left(\operatorname{tr} A^{4}-\operatorname{tr} A^{2}-2 \sum_{j \neq i} a_{i j}^{(2)}\right)$ and $\mathrm{F}=3\left(\operatorname{tr} A^{4}-\operatorname{tr} A^{2}-2\right.$ $\left.\sum_{j \neq i} a_{i j}^{(2)}\right)$. (See Theorem 1.2)

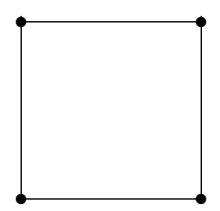

Fig 15

Case 12: For the configuration of Fig 16(a), N=4, M= $\frac{1}{2} \sum_{i=1}^{n}\left(a_{i i}^{(4)}-a_{i i}^{(2)}-2\left(\begin{array}{c}d_{i} \\ 2\end{array}\right)-\sum_{j=1, j \neq i}^{n} a_{i j}^{(2)}\right)\left(d_{i}-2\right)$ (See Theorem 2.1). Let $\mathrm{P}_{1}$ denote the number of all subgraphs of $\mathrm{G}$ that have the same configuration as the graph of Fig 16(b) and are counted in M. Thus $\mathrm{P}_{1}=2 \times \frac{1}{2} \sum_{j \neq i}\left(\begin{array}{c}a_{i j}^{(2)} \\ 2\end{array}\right) a_{i j}$, where $\frac{1}{2} \sum_{j \neq i}\left(\begin{array}{c}a_{i j}^{(2)} \\ 2\end{array}\right) a_{i j}$ is the number of subgraphs of $\mathrm{G}$ that have the same configuration as the graph of Fig 16(b) and 2 is the number of times that this subgraph is counted in M. Consequently, $\mathrm{F}=2 \sum_{i=1}^{n}\left(a_{i i}^{(4)}-a_{i i}^{(2)}-2\left(\begin{array}{c}d_{i} \\ 2\end{array}\right)-\sum_{j=1, j \neq i}^{n} a_{i j}^{(2)}\right)\left(d_{i}-2\right)-4 \sum_{j \neq i}\left(\begin{array}{c}a_{i j}^{(2)} \\ 2\end{array}\right) a_{i j}$.

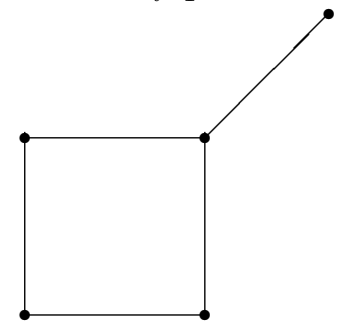

(a)

Fig 16

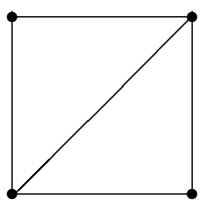

(b)

Case 13: For the configuration of Fig $17, \mathrm{~N}=4, \mathrm{M}=\sum_{j \neq i} a_{i j}^{(2)}\left(\begin{array}{c}d_{i}-a_{i j}-1 \\ 2\end{array}\right)$ and $\mathrm{F}=4 \sum_{j \neq i} a_{i j}^{(2)}\left(\begin{array}{c}d_{i}-a_{i j}-1 \\ 2\end{array}\right)$.

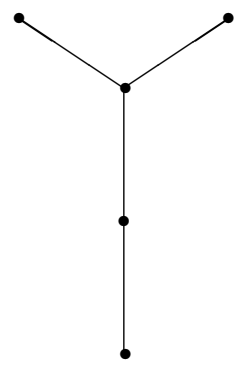

Fig 17

Now we add the values of $\mathrm{F}$ arising from the above cases and determine $x$. Substituting the value of $x$ in $\sum_{j \neq i} a_{i j}^{(5)}-x$ and simplifying, we get the desired result.

Example 2.4 In the graph of Fig 39, $\sum_{j \neq i} a_{i j}^{(5)}=15630, \sum_{j \neq i} a_{i j}^{(4)}=3120, \sum_{i=1}^{6} a_{i i}^{(3)}\left(d_{i}-2\right)=360, \sum_{j \neq i} a_{i j}^{(2)}=120, \sum_{j \neq i} a_{i j}^{(2)}\left(d_{j}-\right.$ $\left.a_{i j}-1\right)=360, \sum_{j \neq i} a_{i j}^{(2)}\left(\begin{array}{c}d_{i}-a_{i j}-1 \\ 2\end{array}\right)=360, \sum_{j \neq i} a_{i j}\left(\begin{array}{c}a_{i j}^{(2)} \\ 2\end{array}\right)=180, \sum_{j \neq i} a_{i i}^{(3)} a_{i j}^{(2)}=2400, \sum_{i=1}^{6} a_{i i}^{(3)}\left(\begin{array}{c}d_{i}-2 \\ 2\end{array}\right)=360$, $\sum_{i=1}^{6}\left(a_{i i}^{(4)}-a_{i i}^{(2)}-2\left(\begin{array}{c}d_{i} \\ 2\end{array}\right)-\sum_{j=1, j \neq i}^{6} a_{i j}^{(2)}\right)\left(d_{i}-2\right)=1080, \sum_{j \neq i} a_{i j}=30, \operatorname{tr} A^{4}=630, \operatorname{tr} A^{3}=120, \operatorname{tr} A^{2}=30$. 
So by Theorem 2.3, the number of paths of length 5 in $K_{6}$ is 720.

Theorem 2.5 Let $G$ be a simple graph with $n$ vertices and the adjacency matrix $A=\left[a_{i j}\right]$. The number of paths of length 5 in $G$, each of which starts from a specific vertex $v_{i}$ is $\sum_{j=1, j \neq i}^{n} a_{i j}^{(5)}-\sum_{j=1, j \neq i}^{n} a_{i j}^{(4)}-\sum_{j=1, j \neq i}^{n} a_{i j}^{(2)}-\sum_{j=1, j \neq i}^{n} a_{i j}-$ $\sum_{j=1, j \neq i}^{n} a_{i j}^{(2)} a_{i j}-2 \sum_{j=1, j \neq i}^{n}\left(\begin{array}{c}d_{j}-1 \\ 2\end{array}\right) a_{i j}^{(2)}+6 \sum_{j=1, j \neq i}^{n}\left(\begin{array}{c}a_{i j}^{(2)} \\ 2\end{array}\right) a_{i j}+\sum_{j=1, j \neq i}^{n} a_{j j}^{(3)} a_{i j}+\sum_{j=1, j \neq i}^{n} a_{i i}^{(3)} a_{i j}-3 \sum_{j=1, j \neq i}^{n} a_{i j}^{(2)}\left(d_{j}-\right.$ $\left.a_{i j}-1\right)-\sum_{j=1, j \neq i}^{n} a_{i j}^{(2)}\left(d_{i}-a_{i j}-1\right)+2 \sum_{j=1, j \neq i}^{n} a_{i j}^{(2)} a_{i j}\left(d_{j}-2\right)-\sum_{j=1, j \neq i}^{n} a_{i i}^{(3)} a_{i j}^{(2)}-\sum_{j=1, j \neq i}^{n} a_{j j}^{(3)} a_{i j}^{(2)}-\sum_{j=1, j \neq i}^{n} a_{i j}^{(2)}\left(d_{j}-a_{i j}-\right.$ $1)\left(d_{i}-1\right)-2 \sum_{j=1, j \neq i}^{n}\left(\frac{1}{2} a_{j j}^{(3)} a_{i j}-a_{i j}^{(2)} a_{i j}\right)\left(d_{j}-3\right)-\left(a_{i i}^{(4)}-a_{i i}^{(2)}-2\left(\begin{array}{c}d_{i} \\ 2\end{array}\right)-\sum_{j=1, j \neq i}^{n} a_{i j}^{(2)}\right)\left(d_{i}-2\right)-\sum_{j=1, j \neq i}^{n}\left(a_{j j}^{(4)}-a_{j j}^{(2)}-\right.$ $\left.2\left(\begin{array}{c}d_{j} \\ 2\end{array}\right)-\sum_{k=1, k \neq j}^{n} a_{j k}^{(2)}\right) a_{i j}-\sum_{j \neq k, j, k \neq i}\left(a_{i j} a_{j k}^{(2)}-a_{i j} a_{i k}\right)\left(d_{j}-2\right)-6\left(\begin{array}{c}d_{i} \\ 2\end{array}\right)-6\left(\begin{array}{c}d_{i} \\ 3\end{array}\right)$.

Proof : By Proposition 1.4, the number of paths of length 5 in a graph G, each of which starts from a specific vertex $v_{i}$ is $\sum_{j=1, j \neq i}^{n} a_{i j}^{(5)}-x$, where $x$ is the number of non-closed walks of length 5 , that begin from $v_{i}$ and are not paths. To find $x$, we have 21 cases as considered below; the cases are based on the configurations-(subgraphs) that generate all non-closed walks of length 5 , each of which starts from the specific vertex $v_{i}$, that are not paths. In each case, $\mathrm{N}$ denote the number of non-closed walks of length 5 , which start from the vertex $v_{i}$ and are not paths in the corresponding subgraph, M denote the number of subgraphs of $\mathrm{G}$ of the same configuration, $\mathrm{F}$ denote the total number of non-closed walks of length 5 , which start from the vertex $v_{i}$ and are not paths in all possible subgraphs of $\mathrm{G}$ of the same configuration. However, in the cases with more than one figure (cases 7, 9,12, 13, 18,19, 20, 21 ), $\mathrm{N}, \mathrm{M}$ and $\mathrm{F}$ are based on the first graph of the respective figures and $\mathrm{P}_{1}, \mathrm{P}_{2}, \ldots$ denote the number of subgraphs of $\mathrm{G}$ which do not have the same configuration as the first graph but are counted in $\mathrm{M}$. It is clear that $\mathrm{F}$ is equal to $\mathrm{N} \times\left(\mathrm{M}-\mathrm{P}_{1}-\mathrm{P}_{2}-\ldots\right)$. To find $\mathrm{N}$ in each case, we have to include in any walk, all the edges and the vertices of the corresponding subgraphs at least once.

Case 1: For the configuration of Fig 18, N=1, M= $\sum_{j=1, j \neq i}^{n} a_{i j}$ and $\mathrm{F}=\sum_{j=1, j \neq i}^{n} a_{i j}$.

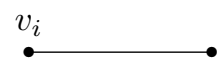

Fig 18

Case 2: For the configuration of Fig 19, $\mathrm{N}=3, \mathrm{M}=\sum_{j=1, j \neq i}^{n} a_{i j}^{(2)}$ and $\mathrm{F}=3 \sum_{j=1, j \neq i}^{n} a_{i j}^{(2)}$.

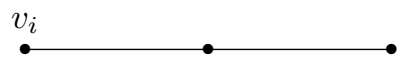

Fig 19

Case 3: For the configuration of Fig 20, $\mathrm{N}=6, \mathrm{M}=\left(\begin{array}{c}d_{i} \\ 2\end{array}\right)$ and $\mathrm{F}=6\left(\begin{array}{c}d_{i} \\ 2\end{array}\right)$.

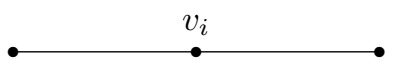

Fig 20

Case 4: For the configuration of Fig 21, $\mathrm{N}=4, \mathrm{M}=\sum_{j=1, j \neq i}^{n} a_{i j}^{(2)}\left(d_{j}-a_{i j}-1\right)$ and $\mathrm{F}=4 \sum_{j=1, j \neq i}^{n} a_{i j}^{(2)}\left(d_{j}-a_{i j}-1\right)$.

(See Theorem 1.8)

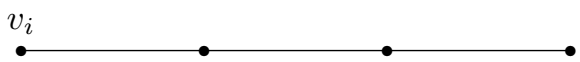

Fig 21 
Case 5: For the configuration of Fig 22, $\mathrm{N}=2, \mathrm{M}=\sum_{j=1, j \neq i}^{n} a_{i j}^{(2)}\left(d_{i}-a_{i j}-1\right)$ and $\mathrm{F}=2 \sum_{j=1, j \neq i}^{n} a_{i j}^{(2)}\left(d_{i}-a_{i j}-1\right)$.

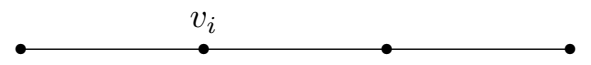

Fig 22

Case 6: For the configuration of Fig $23, \mathrm{~N}=1, \mathrm{M}=\sum_{j=1, j \neq i}^{n}\left[a_{i j}^{(4)}-\left(d_{i}+d_{j}-3 a_{i j}\right) a_{i j}^{(2)}-\left(a_{i i}^{(3)}+a_{j j}^{(3)}+2\left(\begin{array}{c}d_{j}-1 \\ 2\end{array}\right)\right) a_{i j}\right]$ and $\mathrm{F}=\sum_{j=1, j \neq i}^{n}\left[a_{i j}^{(4)}-\left(d_{i}+d_{j}-3 a_{i j}\right) a_{i j}^{(2)}-\left(a_{i i}^{(3)}+a_{j j}^{(3)}+2\left(\begin{array}{c}d_{j}-1 \\ 2\end{array}\right)\right) a_{i j}\right]$. (See Theorem 1.9)

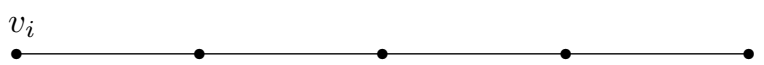

Fig 23

Case 7: For the configuration of Fig 24(a), $\mathrm{N}=1, \mathrm{M}=\sum_{j=1, j \neq i}^{n} a_{i j}^{(2)}\left(d_{j}-a_{i j}-1\right)\left(d_{i}-1\right)$ (See Theorem 1.8). Let $\mathrm{P}_{1}$ denote the number of all subgraphs of $\mathrm{G}$ that have the same configuration as the graph of Fig 24(b) and are counted in M. Thus $\mathrm{P}_{1}=1 \times \sum_{j=1, j \neq i}^{n} a_{i j}^{(2)} a_{i j}\left(d_{j}-2\right)$, where $\sum_{j=1, j \neq i}^{n} a_{i j}^{(2)} a_{i j}\left(d_{j}-2\right)$ is the number of subgraphs of $\mathrm{G}$ that have the same configuration as the graph of Fig 24(b) and this subgraph is counted only once in M. Let $\mathrm{P}_{2}$ denote the number of all subgraphs of $\mathrm{G}$ that have the same configuration as the graph of Fig 24(c) and are counted in M. Thus $\mathrm{P}_{2}=2 \times \frac{1}{2}\left[a_{i i}^{(4)}-a_{i i}^{(2)}-2\left(\begin{array}{c}d_{i} \\ 2\end{array}\right)-\sum_{j=1, j \neq i}^{n} a_{i j}^{(2)}\right]$, where $\frac{1}{2}\left[a_{i i}^{(4)}-a_{i i}^{(2)}-2\left(\begin{array}{c}d_{i} \\ 2\end{array}\right)-\sum_{j=1, j \neq i}^{n} a_{i j}^{(2)}\right]$ is the number of subgraphs of G that have the same configuration as the graph of Fig 24(c) (See Theorem 2.1) and 2 is the number of times that this subgraph is counted in M. Consequently, $\mathrm{F}=\sum_{j=1, j \neq i}^{n} a_{i j}^{(2)}\left(d_{j}-a_{i j}-1\right)\left(d_{i}-1\right)-\sum_{j=1, j \neq i}^{n} a_{i j}^{(2)} a_{i j}\left(d_{j}-\right.$ $2)-\left[a_{i i}^{(4)}-a_{i i}^{(2)}-2\left(\begin{array}{c}d_{i} \\ 2\end{array}\right)-\sum_{j=1, j \neq i}^{n} a_{i j}^{(2)}\right]$.

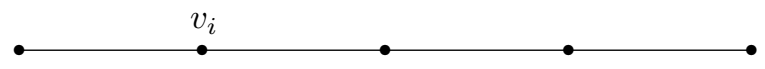

(a)

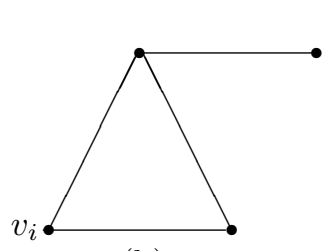

(b)

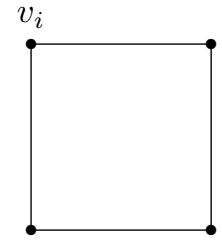

(c)

Fig 24

Case 8: For the configuration of Fig $25, \mathrm{~N}=8, \mathrm{M}=\frac{1}{2} \sum_{j=1, j \neq i}^{n} a_{i j}^{(2)} a_{i j}$ and $\mathrm{F}=4 \sum_{j=1, j \neq i}^{n} a_{i j}^{(2)} a_{i j}$.

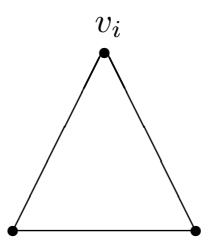

Fig 25 
Case 9: For the configuration of Fig $26, \mathrm{~N}=6, \mathrm{M}=\frac{1}{2} \sum_{j=1, j \neq i}^{n} a_{j j}^{(3)} a_{i j}$. Let $\mathrm{P}_{1}$ denote the number of all subgraphs of G that have the same configuration as the graph of Fig $26(\mathrm{~b})$ and are counted in M. Thus $\mathrm{P}_{1}=2 \times \frac{1}{2} \sum_{j=1, j \neq i}^{n} a_{i j}^{(2)} a_{i j}$, where $\frac{1}{2} \sum_{j=1, j \neq i}^{n} a_{i j}^{(2)} a_{i j}$ is the number of subgraphs of G that have the same configuration as the graph of Fig 26(b) and 2 is the number of times that this subgraph is counted in M. Consequently, $\mathrm{F}=3 \sum_{j=1, j \neq i}^{n} a_{j j}^{(3)} a_{i j}-6 \sum_{j=1, j \neq i}^{n} a_{i j}^{(2)} a_{i j}$.

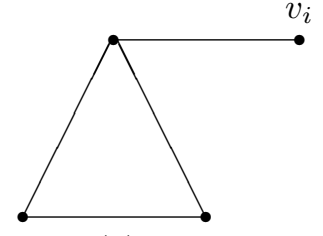

(a)

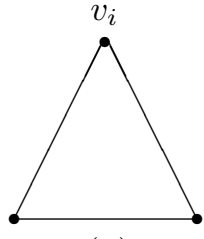

(b)

Case 10: For the configuration of Fig $27, \mathrm{~N}=3, \mathrm{M}=\sum_{j=1, j \neq i}^{n} a_{i j}^{(2)} a_{i j}\left(d_{j}-2\right)$ and $\mathrm{F}=3 \sum_{j=1, j \neq i}^{n} a_{i j}^{(2)} a_{i j}\left(d_{j}-2\right)$.

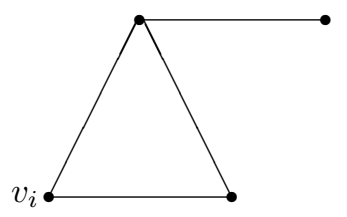

Fig 27

Case 11: For the configuration of Fig $28, \mathrm{~N}=2, \mathrm{M}=\sum_{j=1, j \neq i}^{n}\left(\frac{1}{2} a_{j j}^{(3)} a_{i j}-a_{i j}^{(2)} a_{i j}\right)\left(d_{j}-3\right)$ and $\mathrm{F}=2 \sum_{j=1, j \neq i}^{n}\left(\frac{1}{2} a_{j j}^{(3)} a_{i j}-\right.$ $\left.a_{i j}^{(2)} a_{i j}\right)\left(d_{j}-3\right)$. (See Case 9)

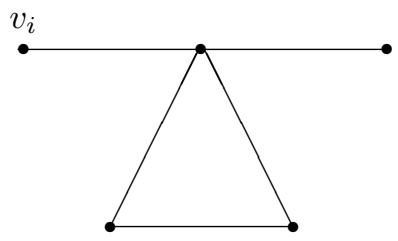

Fig 28

Case 12: For the configuration of Fig $29(a), \mathrm{N}=2, \mathrm{M}=\frac{1}{2} \sum_{j=1, j \neq i}^{n} a_{i i}^{(3)} a_{i j}^{(2)}$ (See Theorem 1.1). Let $\mathrm{P}_{1}$ denote the number of all subgraphs of $\mathrm{G}$ that have the same configuration as the graph of Fig 29(b) and are counted in M. Thus $\mathrm{P}_{1}=2 \times \frac{1}{2} \sum_{j=1, j \neq i}^{n} a_{i j}^{(2)} a_{i j}$, where $\frac{1}{2} \sum_{j=1, j \neq i}^{n} a_{i j}^{(2)} a_{i j}$ is the number of subgraphs of $\mathrm{G}$ that have the same configuration as the graph of Fig 29 (b) and 2 is the number of times that this subgraph is counted in M. Let $\mathrm{P}_{2}$ denote the number of all subgraphs of $\mathrm{G}$ that have the same configuration as the graph of Fig 29(c) and are counted in M. Thus $\mathrm{P}_{2}=1 \times \sum_{j=1, j \neq i}^{n} a_{i j}^{(2)} a_{i j}\left(d_{j}-2\right)$, where $\sum_{j=1, j \neq i}^{n} a_{i j}^{(2)} a_{i j}\left(d_{j}-2\right)$ is the number of subgraphs of G that have the same configuration as the graph of Fig 29(c) and this subgraph is counted only once in M. Let $\mathrm{P}_{3}$ denote the number of all subgraphs of $\mathrm{G}$ that have the same configuration as the graph of Fig 29(d) and are counted in M. Thus $\mathrm{P}_{3}=2 \sum_{j=1, j \neq i}^{n}\left(\begin{array}{c}a_{i j}^{(2)} \\ 2\end{array}\right) a_{i j}$, where $\sum_{j=1, j \neq i}^{n}\left(\begin{array}{c}a_{i j}^{(2)} \\ 2\end{array}\right) a_{i j}$ is the number of subgraphs of $\mathrm{G}$ that have the same configuration as the graph of Fig 29(d) and 2 is the number of times that this subgraph is counted in M. 
Consequently, $\mathrm{F}=\sum_{j=1, j \neq i}^{n} a_{i i}^{(3)} a_{i j}^{(2)}-2 \sum_{j=1, j \neq i}^{n} a_{i j}^{(2)} a_{i j}-2 \sum_{j=1, j \neq i}^{n} a_{i j}^{(2)} a_{i j}\left(d_{j}-2\right)-4 \sum_{j=1, j \neq i}^{n}\left(\begin{array}{c}a_{i j}^{(2)} \\ 2\end{array}\right) a_{i j}$.

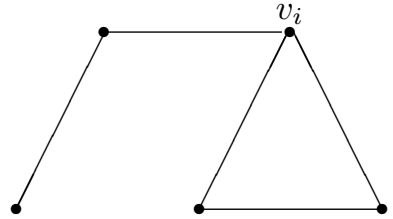

(a)

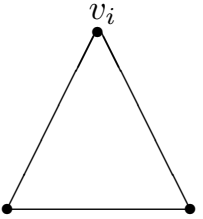

(b)

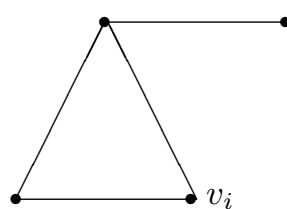

(c)

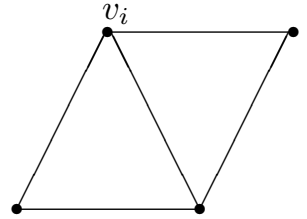

(d)

Fig 29

Case 13: For the configuration of Fig $30(a), \mathrm{N}=2, \mathrm{M}=\frac{1}{2} \sum_{j=1, j \neq i}^{n} a_{i j}^{(2)} a_{j j}^{(3)}$. Let $\mathrm{P}_{1}$ denote the number of all subgraphs of $\mathrm{G}$ that have the same configuration as the graph of Fig 30(b) and are counted in M. Thus $\mathrm{P}_{1}=$ $2 \times \frac{1}{2} \sum_{j=1, j \neq i}^{n} a_{i j}^{(2)} a_{i j}$, where $\frac{1}{2} \sum_{j=1, j \neq i}^{n} a_{i j}^{(2)} a_{i j}$ is the number of subgraphs of $\mathrm{G}$ that have the same configuration as the graph of Fig 30(b) and 2 is the number of times that this subgraph is counted in M. Let $\mathrm{P}_{2}$ denote the number of all subgraphs of $\mathrm{G}$ that have the same configuration as the graph of Fig 30(c) and are counted in M. Thus $\mathrm{P}_{2}=2 \times\left(\frac{1}{2} \sum_{j=1, j \neq i}^{n} a_{j j}^{(3)} a_{i j}-\sum_{j=1, j \neq i}^{n} a_{i j}^{(2)} a_{i j}\right)$, where $\frac{1}{2} \sum_{j=1, j \neq i}^{n} a_{j j}^{(3)} a_{i j}-\sum_{j=1, j \neq i}^{n} a_{i j}^{(2)} a_{i j}$ is the number of subgraphs of $\mathrm{G}$ that have the same configuration as the graph of Fig 30(c) (See Case 9) and 2 is the number of times that this subgraph is counted in $\mathrm{M}$. Let $\mathrm{P}_{3}$ denote the number of all subgraphs of $\mathrm{G}$ that have the same configuration as the graph of Fig 30(d) and are counted in M. Thus $\mathrm{P}_{3}=2 \sum_{j=1, j \neq i}^{n}\left(\begin{array}{c}a_{i j}^{(2)} \\ 2\end{array}\right) a_{i j}$, where $\sum_{j=1, j \neq i}^{n}\left(\begin{array}{c}a_{i j}^{(2)} \\ 2\end{array}\right) a_{i j}$ is the number of subgraphs of $\mathrm{G}$ that have the same configuration as the graph of Fig 30(d) and 2 is the number of times that this subgraph is counted in M. Consequently, $\mathrm{F}=\sum_{j=1, j \neq i}^{n} a_{i j}^{(2)} a_{j j}^{(3)}+2 \sum_{j=1, j \neq i}^{n} a_{i j}^{(2)} a_{i j}-2 \sum_{j=1, j \neq i}^{n} a_{j j}^{(3)} a_{i j}-4 \sum_{j=1, j \neq i}^{n}\left(\begin{array}{c}a_{i j}^{(2)} \\ 2\end{array}\right) a_{i j}$.

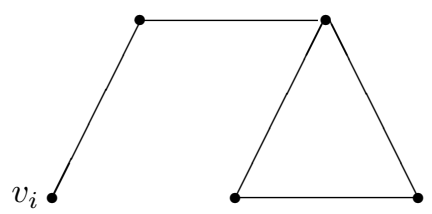

$(a)$

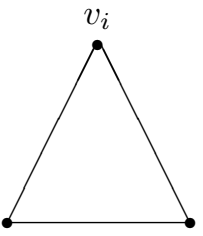

(b)

Fig 30

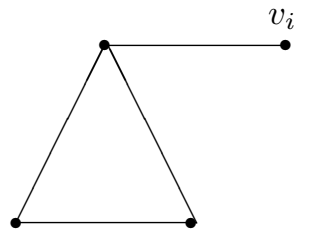

(c)

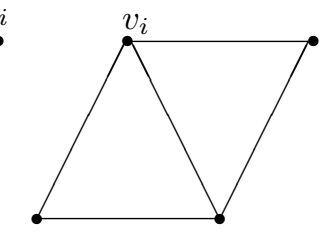

(d)

Case 14: For the configuration of Fig $31, \mathrm{~N}=2, \mathrm{M}=\sum_{j=1, j \neq i}^{n} a_{i j}\left(\begin{array}{c}d_{j}-1 \\ 2\end{array}\right)$ and $\mathrm{F}=2 \sum_{j=1, j \neq i}^{n} a_{i j}\left(\begin{array}{c}d_{j}-1 \\ 2\end{array}\right)$.

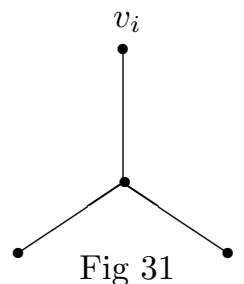

Case 15: For the configuration of Fig $32, \mathrm{~N}=6, \mathrm{M}=\left(\begin{array}{c}d_{i} \\ 3\end{array}\right)$ and $\mathrm{F}=6\left(\begin{array}{c}d_{i} \\ 3\end{array}\right)$. 


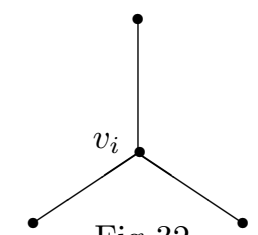

Fig 32

Case 16: For the configuration of Fig $33, \mathrm{~N}=6, \mathrm{M}=\sum_{j=1, j \neq i}^{n}\left(\begin{array}{c}a_{i j}^{(2)} \\ 2\end{array}\right) a_{i j}$ and $\mathrm{F}=6 \sum_{j=1, j \neq i}^{n}\left(\begin{array}{c}a_{i j}^{(2)} \\ 2\end{array}\right) a_{i j}$.

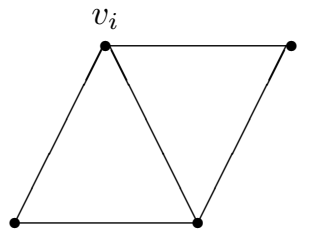

Fig 33

Case 17: For the configuration of Fig $34, \mathrm{~N}=6, \mathrm{M}=\frac{1}{2}\left[a_{i i}^{(4)}-a_{i i}^{(2)}-2\left(\begin{array}{c}d_{i} \\ 2\end{array}\right)-\sum_{j=1, j \neq i}^{n} a_{i j}^{(2)}\right]$ and $\mathrm{F}=3\left[a_{i i}^{(4)}-\right.$ $\left.a_{i i}^{(2)}-2\left(\begin{array}{c}d_{i} \\ 2\end{array}\right)-\sum_{j=1, j \neq i}^{n} a_{i j}^{(2)}\right]$. (See Theorem 2.1)

Fig 34

Case 18: For the configuration of Fig $35(\mathrm{a}), \mathrm{N}=2, \mathrm{M}=\frac{1}{2}\left(a_{i i}^{(4)}-a_{i i}^{(2)}-2\left(\begin{array}{c}d_{i} \\ 2\end{array}\right)-\sum_{j=1, j \neq i}^{n} a_{i j}^{(2)}\right)\left(d_{i}-2\right)$ (See Theorem 2.1). Let $\mathrm{P}_{1}$ denote the number of all subgraphs of $\mathrm{G}$ that have the same configuration as the graph of Fig 35(b) and are counted in M. Thus $\mathrm{P}_{1}=1 \times \sum_{j=1, j \neq i}^{n}\left(\begin{array}{c}a_{i j}^{2} \\ 2\end{array}\right) a_{i j}$, where $\sum_{j=1, j \neq i}^{n}\left(\begin{array}{c}a_{i j}^{2} \\ 2\end{array}\right) a_{i j}$ is the number of subgraphs of $\mathrm{G}$ that have the same configuration as the graph of Fig 35(b) and this subgraph is counted only once in M. Consequently, $\mathrm{F}=\left(a_{i i}^{(4)}-a_{i i}^{(2)}-2\left(\begin{array}{c}d_{i} \\ 2\end{array}\right)-\sum_{j=1, j \neq i}^{n} a_{i j}^{(2)}\right)\left(d_{i}-2\right)-2 \sum_{j=1, j \neq i}^{n}\left(\begin{array}{c}a_{i j}^{(2)} \\ 2\end{array}\right) a_{i j}$.

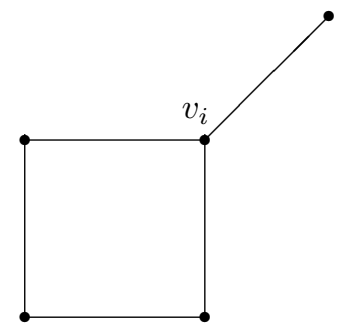

(a)
Fig 35

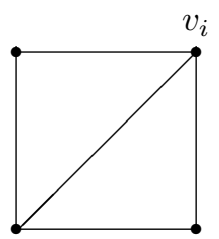

(b)

Case 19: For the configuration of Fig $36(\mathrm{a}), \mathrm{N}=2, \mathrm{M}=\frac{1}{2} \sum_{j=1, j \neq i}^{n}\left(a_{j j}^{(4)}-a_{j j}^{(2)}-2\left(\begin{array}{c}d_{j} \\ 2\end{array}\right)-\sum_{k=1, k \neq j}^{n} a_{j k}^{(2)}\right) a_{i j}($ See Theorem 2.1). Let $\mathrm{P}_{1}$ denote the number of all subgraphs of $\mathrm{G}$ that have the same configuration as the graph of Fig 36(b) and are counted in M. Thus $\mathrm{P}_{1}=1 \times \sum_{j=1, j \neq i}^{n}\left(\begin{array}{c}a_{i j}^{(2)} \\ 2\end{array}\right) a_{i j}$, where $\sum_{j=1, j \neq i}^{n}\left(\begin{array}{c}a_{i j}^{(2)} \\ 2\end{array}\right) a_{i j}$ is the number of subgraphs of $\mathrm{G}$ that have the same configuration as the graph of Fig 36(b) and this subgraph is counted only once in $\mathrm{M}$. Let $\mathrm{P}_{2}$ denote the number of all subgraphs of $\mathrm{G}$ that have the same configuration as the graph of Fig 36(c) and are counted in M. Thus $\mathrm{P}_{2}=2 \times \frac{1}{2}\left[a_{i i}^{(4)}-a_{i i}^{(2)}-2\left(\begin{array}{c}d_{i} \\ 2\end{array}\right)-\sum_{j=1, j \neq i}^{n} a_{i j}^{(2)}\right]$, where $\frac{1}{2}\left[a_{i i}^{(4)}-a_{i i}^{(2)}-2\left(\begin{array}{c}d_{i} \\ 2\end{array}\right)-\sum_{j=1, j \neq i}^{n} a_{i j}^{(2)}\right]$ 
is the number of subgraphs of $\mathrm{G}$ that have the same configuration as the graph of Fig 36(c) (See Theorem 2.1) and 2 is the number of times that this subgraph is counted in M. Consequently, $\mathrm{F}=\sum_{j=1, j \neq i}^{n}\left(a_{j j}^{(4)}-a_{j j}^{(2)}-2\left(\begin{array}{c}d_{j} \\ 2\end{array}\right)\right.$ $\left.-\sum_{k=1, k \neq j}^{n} a_{j k}^{(2)}\right) a_{i j}-2 \sum_{j=1, j \neq i}^{n}\left(\begin{array}{c}a_{i j}^{(2)} \\ 2\end{array}\right) a_{i j}-2\left[a_{i i}^{(4)}-a_{i i}^{(2)}-2\left(\begin{array}{c}d_{i} \\ 2\end{array}\right)-\sum_{j=1, j \neq i}^{n} a_{i j}^{(2)}\right]$.

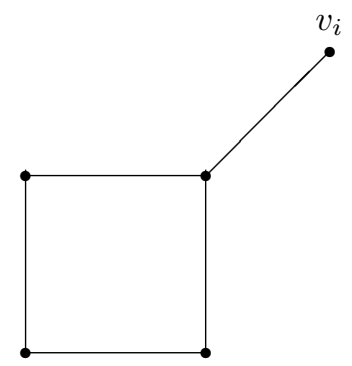

(a)

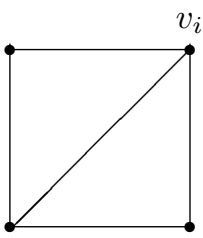

(b)

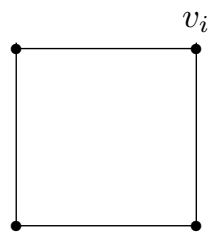

(c)

Fig 36

Case 20: For the configuration of Fig $37(\mathrm{a}), \mathrm{N}=1, \mathrm{M}=\sum_{\substack{j \neq k \\ j, k \neq i}}\left(a_{i j} a_{j k}^{(2)}-a_{i j} a_{i k}\right)\left(d_{j}-2\right)$. Let $\mathrm{P}_{1}$ denote the number of all subgraphs of $\mathrm{G}$ that have the same configuration as the graph of Fig 37(b) and are counted in M. Thus $\mathrm{P}_{1}=2 \times\left[\frac{1}{2} \sum_{j=1, j \neq i}^{n} a_{j j}^{(3)} a_{i j}-\sum_{j=1, j \neq i}^{n} a_{i j}^{(2)} a_{i j}\right]$, where $\frac{1}{2} \sum_{j=1, j \neq i}^{n} a_{j j}^{(3)} a_{i j}-\sum_{j=1, j \neq i}^{n} a_{i j}^{(2)} a_{i j}$ is the number of subgraphs of $\mathrm{G}$ that have the same configuration as the graph of Fig 37(b) (See Case 9) and 2 is the number of times that this subgraph is counted in M. Consequently, $\mathrm{F}=\sum_{\substack{j \neq k \\ j, k \neq i}}\left(a_{i j} a_{j k}^{(2)}-a_{i j} a_{i k}\right)\left(d_{j}-2\right)-\sum_{j=1, j \neq i}^{n} a_{j j}^{(3)} a_{i j}+2 \sum_{j=1, j \neq i}^{n} a_{i j}^{(2)} a_{i j}$.

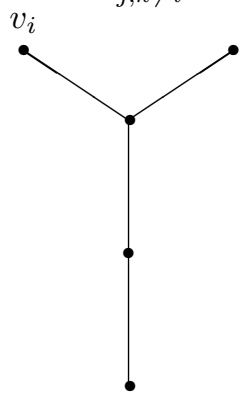

(a)

Fig 37

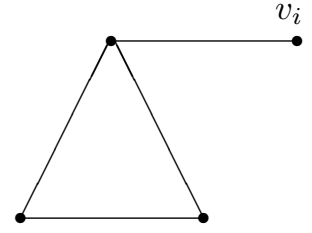

(b)

Case 21: For the configuration of Fig 38(a), $\mathrm{N}=2, \mathrm{M}=\sum_{j=1, j \neq i}^{n} a_{i j}^{(2)}\left(\begin{array}{c}d_{j}-1 \\ 2\end{array}\right)$. Let $\mathrm{P}_{1}$ denote the number of all subgraphs of $\mathrm{G}$ that have the same configuration as the graph of Fig 38(b) and are counted in M. Thus $\mathrm{P}_{1}=1 \times \sum_{j=1, j \neq i}^{n} a_{i j}^{(2)} a_{i j}\left(d_{j}-2\right)$, where $\sum_{j=1, j \neq i}^{n} a_{i j}^{(2)} a_{i j}\left(d_{j}-2\right)$ is the number of subgraphs of $\mathrm{G}$ that have the same configuration as the graph of Fig 38(b) and this subgraph is counted only once in M. Consequently, F= $2 \sum_{j=1, j \neq i}^{n} a_{i j}^{(2)}\left(\begin{array}{c}d_{j}-1 \\ 2\end{array}\right)-2 \sum_{j=1, j \neq i}^{n} a_{i j}^{(2)} a_{i j}\left(d_{j}-2\right)$. 


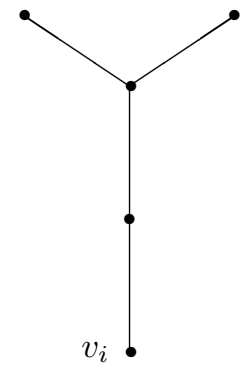

(a)

Fig 38

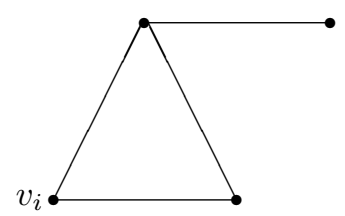

(b)

Now we add the values of $\mathrm{F}$ arising from the above cases and determine $x$. Substituting the value of $x$ in $\sum_{j=1, j \neq i} a_{i j}^{(5)}-x$ and simplifying, we get the desired result.

Example 2.6 In the graph of Fig 39, $\sum_{j=2}^{6} a_{1 j}^{(5)}=2605, \sum_{j=2}^{6} a_{1 j}^{(4)}=520, \sum_{j=2}^{6} a_{1 j}^{(2)}=20, \sum_{j=2}^{6} a_{1 j}=5, \sum_{j=2}^{6} a_{1 j}^{(2)} a_{1 j}=20$, $\sum_{j=2}^{6}\left(\begin{array}{c}d_{j}-1 \\ 2\end{array}\right) a_{1 j}^{(2)}=120, \sum_{j=2}^{6}\left(\begin{array}{c}a_{1 j}^{(2)} \\ 2\end{array}\right) a_{1 j}=30, \sum_{j=2}^{6} a_{j j}^{(3)} a_{1 j}=100, \sum_{j=2}^{6} a_{11}^{(3)} a_{1 j}=100, \sum_{j=2}^{6} a_{1 j}^{(2)}\left(d_{j}-a_{1 j}-1\right)=$ $60, \sum_{j=2}^{6} a_{1 j}^{(2)}\left(d_{1}-a_{1 j}-1\right)=60, \sum_{j=2}^{6} a_{1 j}^{(2)} a_{1 j}\left(d_{j}-2\right)=60, \sum_{j=2}^{6} a_{11}^{(3)} a_{1 j}^{(2)}=400, \sum_{j=2}^{6} a_{j j}^{(3)} a_{1 j}^{(2)}=400, \sum_{j=2}^{6} a_{1 j}^{(2)}\left(d_{j}-\right.$ $\left.a_{1 j}-1\right)\left(d_{1}-1\right)=240, \sum_{j=2}^{6}\left(\frac{1}{2} a_{j j}^{(3)} a_{1 j}-a_{1 j}^{(2)} a_{1 j}\right)\left(d_{j}-3\right)=60,\left(a_{11}^{(4)}-a_{11}^{(2)}-2\left(\begin{array}{c}d_{1} \\ 2\end{array}\right)-\sum_{j=2}^{6} a_{1 j}^{(2)}\right)\left(d_{1}-2\right)=180$, $\sum_{j=2}^{6}\left(a_{j j}^{(4)}-a_{j j}^{(2)}-2\left(\begin{array}{c}d_{j} \\ 2\end{array}\right)-\sum_{k=1, k \neq j}^{6} a_{j k}^{(2)}\right) a_{1 j}=300, \sum_{j \neq k, j, k \neq 1}\left(a_{1 j} a_{j k}^{(2)}-a_{1 j} a_{1 k}\right)\left(d_{j}-2\right)=180,\left(\begin{array}{c}d_{1} \\ 2\end{array}\right)=10,\left(\begin{array}{c}d_{1} \\ 3\end{array}\right)=$ 10. So, by Theorem 2.5, the number of paths of length 5, starting from the vertex $v_{1}$ in the graph of Fig 39 is 120.

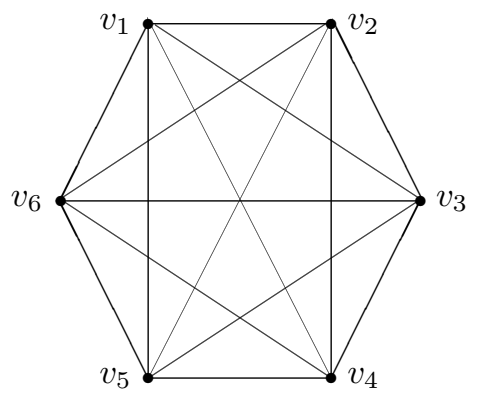

Fig 39

Theorem 2.7 Let $G$ be a simple graph with $n$ vertices and the adjacency matrix $A=\left[a_{i j}\right]$. The number of $v_{i}-v_{j}$ paths of length 5 in $G$ is $a_{i j}^{(5)}-\left(2 d_{i}+2 d_{j}+d_{i} d_{j}+a_{i i}^{(4)}+a_{j j}^{(4)}-a_{i i}^{(2)}-a_{j j}^{(2)}-a_{i j}^{(2)}-2\left(\begin{array}{c}d_{i} \\ 2\end{array}\right)-2\left(\begin{array}{c}d_{j} \\ 2\end{array}\right)+2\left(\begin{array}{c}d_{i}-1 \\ 2\end{array}\right)\right.$ $\left.+2\left(\begin{array}{c}d_{j}-1 \\ 2\end{array}\right)-6\left(\begin{array}{c}a_{i j}^{(2)} \\ 2\end{array}\right)-4\right) a_{i j}-\left(a_{i i}^{(3)}+a_{j j}^{(3)}\right) a_{i j}^{(2)}+\sum_{k=1, k \neq i}^{n} a_{i k}^{(2)} a_{i j}+\sum_{k=1, k \neq j}^{n} a_{j k}^{(2)} a_{i j}-\sum_{k=1, k \neq i, j}^{n} a_{k k}^{(3)} a_{i k} a_{j k}$ $-\sum_{k=1, k \neq i, j}^{n}\left(a_{i k}^{(2)}+a_{j k}^{(2)}-a_{i k}-a_{j k}-2 a_{i k} a_{j k}\right) a_{i j}+\sum_{k=1, k \neq i, j}^{n}\left(a_{i k}^{(2)}-a_{i j}\right)\left(3 a_{i j}+3 a_{i k}-d_{i}-d_{j}-d_{k}+1\right) a_{j k}$ $+\sum_{k=1, k \neq i, j}^{n}\left(a_{j k}^{(2)}-a_{i j}\right)\left(3 a_{j k}-d_{k}+2\right) a_{i k}$.

Proof: By Proposition 1.5, the number of $v_{i}-v_{j}(j \neq i)$ paths of length 5 in a graph $\mathrm{G}$ is $a_{i j}^{(5)}-x$, where $x$ is the number of $v_{i}-v_{j}(j \neq i)$ walks of length 5 , that are not paths. To find $x$, we have 23 cases as considered below; the cases are based on the configurations-(subgraphs) that generate all $v_{i}-v_{j}(j \neq i)$ walks of length 5 , that are not 
paths. In each case, $\mathrm{N}$ denote the number of $v_{i}-v_{j}(j \neq i)$ walks of length 5 , that are not paths in the corresponding subgraph, $\mathrm{M}$ denote the number of subgraphs of $\mathrm{G}$ of the same configuration, $\mathrm{F}$ denote the total number of $v_{i}-v_{j}$ $(j \neq i)$ walks of length 5 that are not paths in all possible subgraphs of $\mathrm{G}$ of the same configuration. However, in the cases with more than one figure (Cases 5, 6, 7, 8, 9, 10, 11, 14, 15, 17, 18, 19, 22 and 23), N, M and F are based on the first graph of the respective figures and $\mathrm{P}_{1}, \mathrm{P}_{2}, \ldots$ denote the number of subgraphs of $\mathrm{G}$ which do not have the same configuration as the first graph but are counted in $\mathrm{M}$. It is clear that $\mathrm{F}$ is equal to $\mathrm{N} \times\left(\mathrm{M}-\mathrm{P}_{1}-\mathrm{P}_{2}-\ldots\right)$. To find $\mathrm{N}$ in each case, we have to include in any walk, all the edges and the vertices of the corresponding subgraphs at least once.

Case 1: For the configuration of Fig $40, \mathrm{~N}=1, \mathrm{M}=a_{i j}$ and $\mathrm{F}=a_{i j}$.

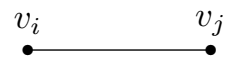

Fig 40

Case 2: For the configuration of Fig $41, \mathrm{~N}=3, \mathrm{M}=a_{i j}\left(d_{j}-1\right)$ and $\mathrm{F}=3 a_{i j}\left(d_{j}-1\right)$.

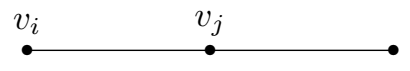

Fig 41

Case 3: For the configuration of Fig $42, \mathrm{~N}=3, \mathrm{M}=a_{i j}\left(d_{i}-1\right)$ and $\mathrm{F}=3 a_{i j}\left(d_{i}-1\right)$.

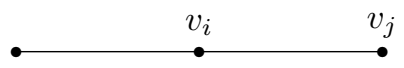

Fig 42

Case 4: For the configuration of Fig 43, N=3, M= $\sum_{k=1, k \neq i, j}^{n}\left(a_{i k}^{(2)}-a_{i j}\right) a_{j k}$ and $\mathrm{F}=3 \sum_{k=1, k \neq i, j}^{n}\left(a_{i k}^{(2)}-a_{i j}\right) a_{j k}$.

(See Theorem 1.10)

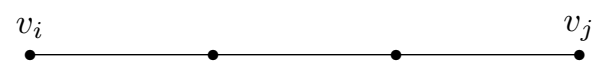

Fig 43

Case 5: For the configuration of Fig $44(\mathrm{a}), \mathrm{N}=1, \mathrm{M}=a_{i j}\left(d_{i}-1\right)\left(d_{j}-1\right)$. Let $\mathrm{P}_{1}$ denote the number of subgraphs of $\mathrm{G}$ that have the same configuration as the graph of Fig $44(\mathrm{~b})$ and are counted in $\mathrm{M}$. Thus $\mathrm{P}_{1}=1 \times a_{i j}^{(2)} a_{i j}$, where $a_{i j}^{(2)} a_{i j}$ is the number of subgraphs of $\mathrm{G}$ that have the same configuration as the graph of Fig 44(b) and this subgraph is counted only once in M. Consequently, $\mathrm{F}=a_{i j}\left(d_{i}-1\right)\left(d_{j}-1\right)-a_{i j}^{(2)} a_{i j}$.

(a)

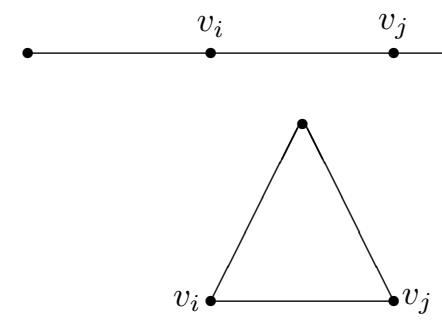

Fig 44

Case 6: For the configuration of Fig $45(\mathrm{a}), \mathrm{N}=1, \mathrm{M}=\sum_{k=1, k \neq i, j}^{n} a_{i j} a_{j k}^{(2)}$. Let $\mathrm{P}_{1}$ denote the number of subgraphs of $\mathrm{G}$ that have the same configuration as the graph of Fig $45(\mathrm{~b})$ and are counted in M. Thus $\mathrm{P}_{1}=1 \times \sum_{k=1, k \neq i, j}^{n} a_{i j} a_{i k}$, where $\sum_{k=1, k \neq i, j}^{n} a_{i j} a_{i k}$ is the number of subgraphs of $\mathrm{G}$ that have the same configuration as the graph of Fig $45(\mathrm{~b})$ and this subgraph is counted only once in M. Consequently, $\mathrm{F}=\sum_{k=1, k \neq i, j}^{n} a_{i j} a_{j k}^{(2)}-\sum_{k=1, k \neq i, j}^{n} a_{i j} a_{i k}$. 

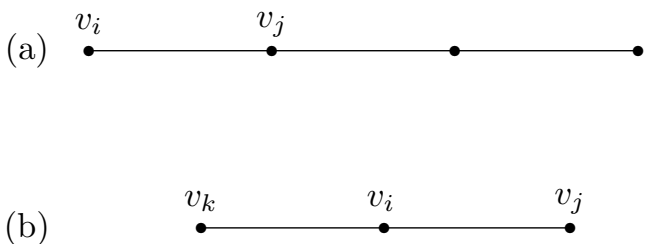

Fig 45

Case 7: For the configuration of Fig 46(a), $\mathrm{N}=1, \mathrm{M}=\sum_{k=1, k \neq i, j}^{n} a_{i j} a_{i k}^{(2)}$. Let $\mathrm{P}_{1}$ denote the number of subgraphs of $\mathrm{G}$ that have the same configuration as the graph of Fig 46(b) and are counted in M. Thus $\mathrm{P}_{1}=1 \times \sum_{k=1, k \neq i, j}^{n} a_{i j} a_{j k}$, where $\sum_{k=1, k \neq i, j}^{n} a_{i j} a_{j k}$ is the number of subgraphs of G that have the same configuration as the graph of Fig 46(b) and this subgraph is counted only once in M. Consequently, $\mathrm{F}=\sum_{k=1, k \neq i, j}^{n} a_{i j} a_{i k}^{(2)}-\sum_{k=1, k \neq i, j}^{n} a_{i j} a_{j k}$.

(a)

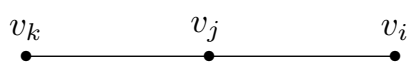

Fig 46

Case 8: For the configuration of Fig $47(\mathrm{a}), \mathrm{N}=3, \mathrm{M}=\sum_{k=1, k \neq i, j}^{n} a_{j k}^{(2)} a_{j k} a_{i k}$. Let $\mathrm{P}_{1}$ denote the number of subgraphs of $\mathrm{G}$ that have the same configuration as the graph of Fig $47(\mathrm{~b})$ and are counted in M. Thus $\mathrm{P}_{1}=1 \times \sum_{k=1, k \neq i, j}^{n} a_{i k} a_{i j} a_{j k}$, where $\sum_{k=1, k \neq i, j}^{n} a_{i k} a_{i j} a_{j k}$ is the number of subgraphs of G that have the same configuration as the graph of Fig 47(b) and this subgraph is counted only once in M. Consequently, $\mathrm{F}=3 \sum_{k=1, k \neq i, j}^{n}\left(a_{j k}^{(2)}-a_{i j}\right) a_{i k} a_{j k}$.

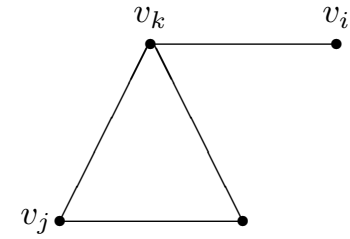

(a)

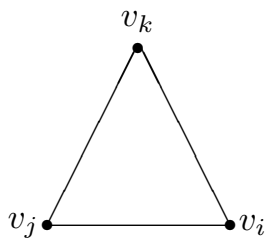

(b)

Fig 47

Case 9: For the configuration of Fig 48(a), $\mathrm{N}=3, \mathrm{M}=\sum_{k=1, k \neq i, j}^{n} a_{i k}^{(2)} a_{i k} a_{j k}$. Let $\mathrm{P}_{1}$ denote the number of subgraphs of $\mathrm{G}$ that have the same configuration as the graph of Fig 48(b) and are counted in M. Thus $\mathrm{P}_{1}=1 \times \sum_{k=1, k \neq i, j}^{n} a_{i k} a_{i j} a_{j k}$, where $\sum_{k=1, k \neq i, j}^{n} a_{i k} a_{i j} a_{j k}$ is the number of subgraphs of G that have the same configuration as the graph of Fig 48(b) and this subgraph is counted only once in M. Consequently,

$\mathrm{F}=3 \sum_{k=1, k \neq i, j}^{n}\left(a_{i k}^{(2)}-a_{i j}\right) a_{i k} a_{j k}$. 


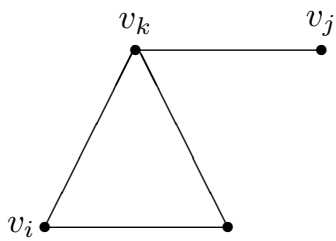

(a)

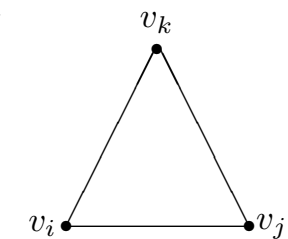

(b)

Fig 48

Case 10: For the configuration of Fig 49(a), $\mathrm{N}=1, \mathrm{M}=\sum_{k=1, k \neq i, j}^{n}\left(a_{i k}^{(2)}-a_{i j}\right)\left(d_{j}-1\right) a_{j k}$ (See Theorem 1.10). Let $\mathrm{P}_{1}$ denote the number of subgraphs of $\mathrm{G}$ that have the same configuration as the graph of Fig 49(b) and are counted in M. Thus $\mathrm{P}_{1}=1 \times \sum_{k=1, k \neq i, j}^{n}\left(a_{j k}^{(2)}-a_{i j}\right) a_{i k} a_{j k}$ (See Case 8), where $\sum_{k=1, k \neq i, j}^{n}\left(a_{j k}^{(2)}-a_{i j}\right) a_{i k} a_{j k}$ is the number of subgraphs of $\mathrm{G}$ that have the same configuration as the graph of Fig 49(b) and this subgraph is counted only once in $\mathrm{M}$. Let $\mathrm{P}_{2}$ denote the number of subgraphs of $\mathrm{G}$ that have the same configuration as the graph of Fig $49(\mathrm{c})$ and are counted in M. Thus $\mathrm{P}_{2}=1 \times \sum_{k=1, k \neq i, j}^{n}\left(a_{i k}^{(2)}-a_{i j}\right) a_{j k} a_{i j}$ (See Theorem 1.10), where $\sum_{k=1, k \neq i, j}^{n}\left(a_{i k}^{(2)}-a_{i j}\right) a_{j k} a_{i j}$ is the number of subgraphs of $\mathrm{G}$ that have the same configuration as the graph of Fig $49(\mathrm{c})$ and this subgraph is counted only once in M. Consequently, $\mathrm{F}=\sum_{k=1, k \neq i, j}^{n}\left(a_{i k}^{(2)}-a_{i j}\right)\left(d_{j}-a_{i j}-1\right) a_{j k}-\sum_{k=1, k \neq i, j}^{n}\left(a_{j k}^{(2)}-a_{i j}\right) a_{i k} a_{j k}$.

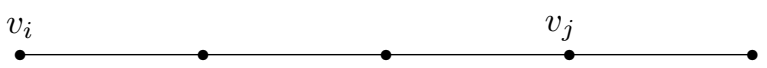

(a)

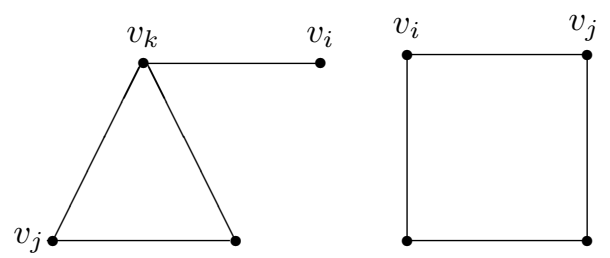

(b) $\quad$ Fig $49 \quad$ (c)

Case 11: For the configuration of Fig $50(\mathrm{a}), \mathrm{N}=1, \mathrm{M}=\sum_{k=1, k \neq i, j}^{n}\left(a_{i k}^{(2)}-a_{i j}\right)\left(d_{i}-1\right) a_{j k}$ (See Theorem 1.10). Let $\mathrm{P}_{1}$ denote the number of subgraphs of $\mathrm{G}$ that have the same configuration as the graph of Fig 50(b) and are counted in M. Thus $\mathrm{P}_{1}=1 \times \sum_{k=1, k \neq i, j}^{n}\left(a_{i k}^{(2)}-a_{i j}\right) a_{i k} a_{j k}$ (See Case 9), where $\sum_{k=1, k \neq i, j}^{n}\left(a_{i k}^{(2)}-a_{i j}\right) a_{i k} a_{j k}$ is the number of subgraphs of $\mathrm{G}$ that have the same configuration as the graph of Fig 50(b) and this subgraph is counted only once in $\mathrm{M}$. Let $\mathrm{P}_{2}$ denote the number of subgraphs of $\mathrm{G}$ that have the same configuration as the graph of Fig 50(c) and are counted in M. Thus $\mathrm{P}_{2}=1 \times \sum_{k=1, k \neq i, j}^{n}\left(a_{i k}^{(2)}-a_{i j}\right) a_{j k} a_{i j}$ (See Theorem 1.10), where $\sum_{k=1, k \neq i, j}^{n}\left(a_{i k}^{(2)}-a_{i j}\right) a_{j k} a_{i j}$ is the number of subgraphs of $\mathrm{G}$ that have the same configuration as the graph of Fig $50(\mathrm{c})$ and this subgraph is counted only once in M. Consequently, $\mathrm{F}=\sum_{k=1, k \neq i, j}^{n}\left(a_{i k}^{(2)}-a_{i j}\right)\left(d_{i}-a_{i j}-1\right) a_{j k}-\sum_{k=1, k \neq i, j}^{n}\left(a_{i k}^{(2)}-a_{i j}\right) a_{i k} a_{j k}$.

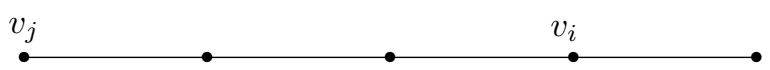

(a)

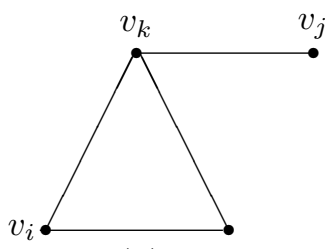

(b)

Fig 50

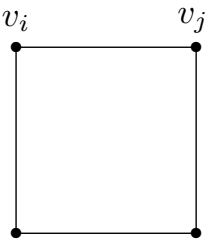

(c) 
Case 12: For the configuration of Fig $51, \mathrm{~N}=2, \mathrm{M}=a_{i j}\left(\begin{array}{c}d_{j}-1 \\ 2\end{array}\right)$ and $\mathrm{F}=2 a_{i j}\left(\begin{array}{c}d_{j}-1 \\ 2\end{array}\right)$.

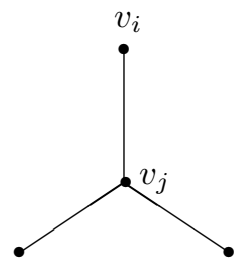

Fig 51

Case 13: For the configuration of Fig $52, \mathrm{~N}=2, \mathrm{M}=a_{i j}\left(\begin{array}{c}d_{i}-1 \\ 2\end{array}\right)$ and $\mathrm{F}=2 a_{i j}\left(\begin{array}{c}d_{i}-1 \\ 2\end{array}\right)$.

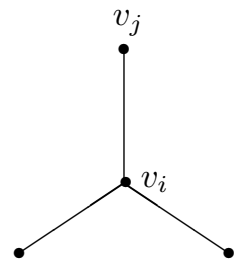

Fig 52

Case 14: For the configuration of Fig $53(\mathrm{a}), \mathrm{N}=1, \mathrm{M}=\sum_{k=1, k \neq i, j}^{n}\left(a_{j k}^{(2)}-a_{i j}\right)\left(d_{k}-2\right) a_{i k}$ (See Theorem 1.10). Let $\mathrm{P}_{1}$ denote the number of subgraphs of $\mathrm{G}$ that have the same configuration as the graph of Fig 53(b) and are counted in M. Thus $\mathrm{P}_{1}=1 \times \sum_{k=1, k \neq i, j}^{n}\left(a_{j k}^{(2)}-a_{i j}\right) a_{j k} a_{i k}$, where $\sum_{k=1, k \neq i, j}^{n}\left(a_{j k}^{(2)}-a_{i j}\right) a_{j k} a_{i k}$ is the number of subgraphs of $\mathrm{G}$ that have the same configuration as the graph of Fig 53(b) (See Case 8) and this subgraph is counted only once in M. Consequently, $\mathrm{F}=\sum_{k=1, k \neq i, j}^{n}\left(a_{j k}^{(2)}-a_{i j}\right)\left(d_{k}-a_{j k}-2\right) a_{i k}$.

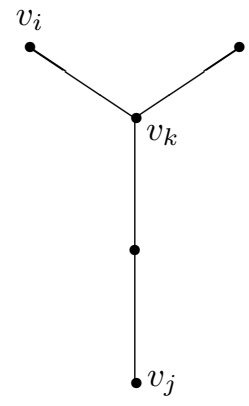

(a)

Fig 53

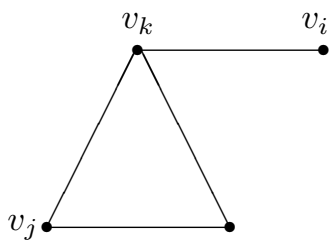

(b)

Case 15: For the configuration of Fig $54(\mathrm{a}), \mathrm{N}=1, \mathrm{M}=\sum_{k=1, k \neq i, j}^{n}\left(a_{i k}^{(2)}-a_{i j}\right)\left(d_{k}-2\right) a_{j k}$ (See Theorem 1.10). Let $\mathrm{P}_{1}$ denote the number of subgraphs of $\mathrm{G}$ that have the same configuration as the graph of Fig 54(b) and are counted in M. Thus $\mathrm{P}_{1}=1 \times \sum_{k=1, k \neq i, j}^{n}\left(a_{i k}^{(2)}-a_{i j}\right) a_{i k} a_{j k}$, where $\sum_{k=1, k \neq i, j}^{n}\left(a_{i k}^{(2)}-a_{i j}\right) a_{i k} a_{j k}$ is the number of subgraphs of $\mathrm{G}$ that have the same configuration as the graph of Fig 54(b) (See Case 9) and this subgraph is counted only once in M. Consequently, $\mathrm{F}=\sum_{k=1, k \neq i, j}^{n}\left(a_{i k}^{(2)}-a_{i j}\right)\left(d_{k}-a_{i k}-2\right) a_{j k}$. 


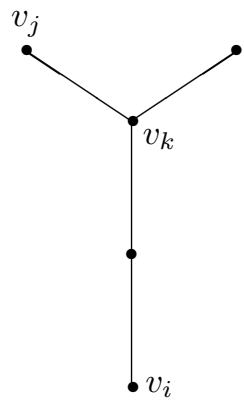

(a)

Fig 54

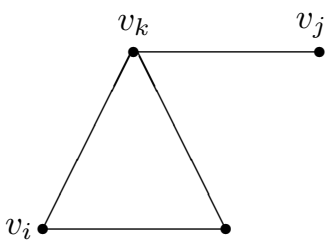

(b)

Case 16: For the configuration of Fig $55, \mathrm{~N}=4, \mathrm{M}=a_{i j}^{(2)} a_{i j}$ and $\mathrm{F}=4 a_{i j}^{(2)} a_{i j}$.

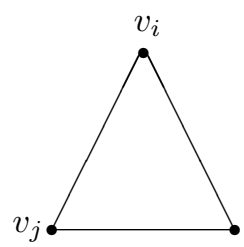

Fig 55

Case 17: For the configuration of Fig 56(a), $\mathrm{N}=2, \mathrm{M}=\frac{1}{2} a_{i i}^{(3)} a_{i j}^{(2)}$. Let $\mathrm{P}_{1}$ denote the number of subgraphs of $\mathrm{G}$ that have the same configuration as the graph of Fig 56(b) and are counted in M. Thus $\mathrm{P}_{1}=1 \times a_{i j}^{(2)} a_{i j}$, where $a_{i j}^{(2)} a_{i j}$ is the number of subgraphs of $\mathrm{G}$ that have the same configuration as the graph of Fig $56(\mathrm{~b})$ and this subgraph is counted only once in $\mathrm{M}$. Let $\mathrm{P}_{2}$ denote the number of subgraphs of $\mathrm{G}$ that have the same configuration as the graph of Fig 56(c) and are counted in M. Thus $\mathrm{P}_{2}=1 \times \sum_{k=1, k \neq i, j}^{n}\left(a_{i k}^{(2)}-a_{i j}\right) a_{i k} a_{j k}$, where $\sum_{k=1, k \neq i, j}^{n}\left(a_{i k}^{(2)}-a_{i j}\right) a_{i k} a_{j k}$ is the number of subgraphs of $\mathrm{G}$ that have the same configuration as the graph of Fig 56(c) (See Case 9) and this subgraph is counted only once in $\mathrm{M}$. Let $\mathrm{P}_{3}$ denote the number of subgraphs of $\mathrm{G}$ that have the same configuration as the graph of Fig 56(d) and are counted in M. Thus $\mathrm{P}_{3}=2 \times\left(\begin{array}{c}a_{i j}^{(2)} \\ 2\end{array}\right) a_{i j}$, where $\left(\begin{array}{c}a_{i j}^{(2)} \\ 2\end{array}\right) a_{i j}$ is the number of subgraphs of G that have the same configuration as the graph of Fig 56(d) and 2 is the number of times that this subgraph is counted in M. Consequently,

$\mathrm{F}=a_{i i}^{(3)} a_{i j}^{(2)}-2 a_{i j}^{(2)} a_{i j}-4\left(\begin{array}{c}a_{i j}^{(2)} \\ 2\end{array}\right) a_{i j}-2 \sum_{k=1, k \neq i, j}^{n}\left(a_{i k}^{(2)}-a_{i j}\right) a_{i k} a_{j k}$.

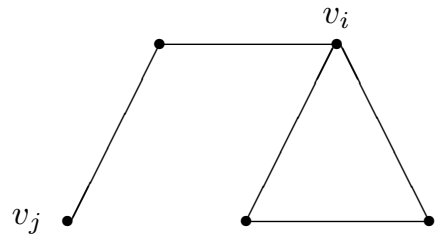

(a)

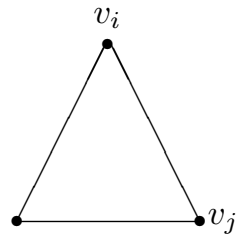

$(b)$

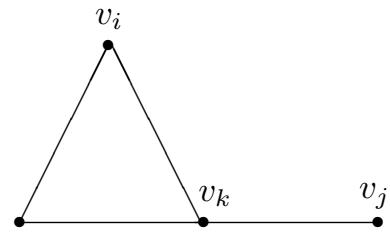

$(c)$

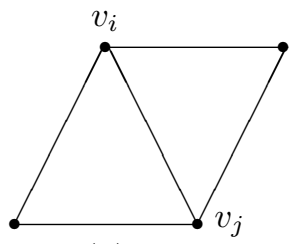

$(d)$

Fig 56

Case 18: For the configuration of Fig $57(\mathrm{a}), \mathrm{N}=2, \mathrm{M}=\frac{1}{2} a_{j j}^{(3)} a_{i j}^{(2)}$. Let $\mathrm{P}_{1}$ denote the number of subgraphs of $\mathrm{G}$ that have the same configuration as the graph of Fig $57(\mathrm{~b})$ and are counted in M. Thus $\mathrm{P}_{1}=1 \times a_{i j}^{(2)} a_{i j}$, where $a_{i j}^{(2)} a_{i j}$ is the number of subgraphs of $\mathrm{G}$ that have the same configuration as the graph of Fig $57(\mathrm{~b})$ and this subgraph is counted only once in $\mathrm{M}$. Let $\mathrm{P}_{2}$ denote the number of subgraphs of $\mathrm{G}$ that have the same configuration as the graph of Fig 57(c) and are counted in M. Thus $\mathrm{P}_{2}=1 \times \sum_{k=1, k \neq i, j}^{n}\left(a_{j k}^{(2)}-a_{i j}\right) a_{i k} a_{j k}$, where $\sum_{k=1, k \neq i, j}^{n}\left(a_{j k}^{(2)}-a_{i j}\right) a_{i k} a_{j k}$ is the number of subgraphs of $\mathrm{G}$ that have the same configuration as the graph of Fig 57(c) (See Case 8) and this subgraph is counted only once in $\mathrm{M}$. Let $\mathrm{P}_{3}$ denote the number of subgraphs of $\mathrm{G}$ that have the same configuration 
as the graph of Fig $57(\mathrm{~d})$ and are counted in M. Thus $\mathrm{P}_{3}=2 \times\left(\begin{array}{c}a_{i j}^{(2)} \\ 2\end{array}\right) a_{i j}$, where $\left(\begin{array}{c}a_{i j}^{(2)} \\ 2\end{array}\right) a_{i j}$ is the number of subgraphs of $\mathrm{G}$ that have the same configuration as the graph of Fig $57(\mathrm{~d})$ and 2 is the number of times that this subgraph is counted in M. Consequently,

$\mathrm{F}=a_{j j}^{(3)} a_{i j}^{(2)}-2 a_{i j}^{(2)} a_{i j}-4\left(\begin{array}{c}a_{i j}^{(2)} \\ 2\end{array}\right) a_{i j}-2 \sum_{k=1, k \neq i, j}^{n}\left(a_{j k}^{(2)}-a_{i j}\right) a_{i k} a_{j k}$.

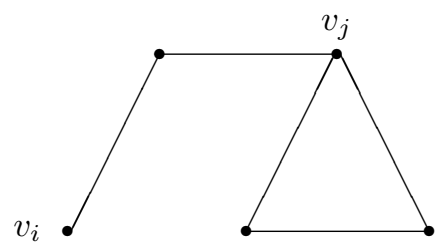

(a)

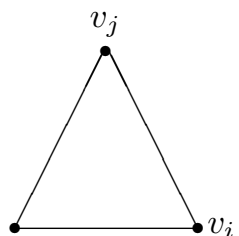

(b)

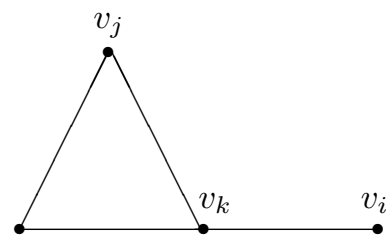

$(c)$

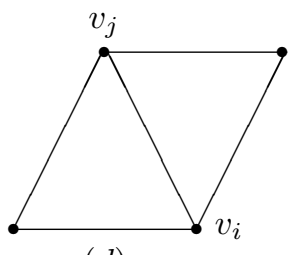

$(d)$

Fig 57

Case 19: For the configuration of Fig 58(a), N=2, M= $\frac{1}{2} \sum_{k=1, k \neq i, j}^{n} a_{k k}^{(3)} a_{i k} a_{j k}$. Let $\mathrm{P}_{1}$ denote the number of subgraphs of $\mathrm{G}$ that have the same configuration as the graph of Fig 58(b) and are counted in $\mathrm{M}$. Thus $\mathrm{P}_{1}=$ $1 \times \sum_{k=1, k \neq i, j}^{n}\left(a_{i k}^{(2)}-a_{i j}\right) a_{i k} a_{j k}$, where $\sum_{k=1, k \neq i, j}^{n}\left(a_{i k}^{(2)}-a_{i j}\right) a_{i k} a_{j k}$ is the number of subgraphs of $\mathrm{G}$ that have the same configuration as the graph of Fig 58(b) (See Case 9) and this subgraph is counted only once in M. Let $\mathrm{P}_{2}$ denote the number of subgraphs of $\mathrm{G}$ that have the same configuration as the graph of Fig 58(c) and are counted in M. Thus $\mathrm{P}_{2}=1 \times \sum_{k=1, k \neq i, j}^{n}\left(a_{j k}^{(2)}-a_{i j}\right) a_{i k} a_{j k}$, where $\sum_{k=1, k \neq i, j}^{n}\left(a_{j k}^{(2)}-a_{i j}\right) a_{i k} a_{j k}$ is the number of subgraphs of $\mathrm{G}$ that have the same configuration as the graph of Fig 58(c) (See Case 8) and this subgraph is counted only once in M. Let $\mathrm{P}_{3}$ denote the number of subgraphs of $\mathrm{G}$ that have the same configuration as the graph of Fig 58(d) and are counted in M. Thus $\mathrm{P}_{3}=1 \times \sum_{k=1, k \neq i, j}^{n} a_{i j} a_{j k} a_{i k}$, where $\sum_{k=1, k \neq i, j}^{n} a_{i j} a_{j k} a_{i k}$ is the number of subgraphs of $\mathrm{G}$ that have the same configuration as the graph of Fig 58(d) and this subgraph is counted only once in M. Consequently, F= $\sum_{k=1, k \neq i, j}^{n} a_{k k}^{(3)} a_{i k} a_{j k}-2 \sum_{k=1, k \neq i, j}^{n}\left(a_{i k}^{(2)}-a_{i j}\right) a_{i k} a_{j k}-2 \sum_{k=1, k \neq i, j}^{n}\left(a_{j k}^{(2)}-a_{i j}\right) a_{i k} a_{j k}-2 \sum_{k=1, k \neq i, j}^{n} a_{i j} a_{j k} a_{i k}$.

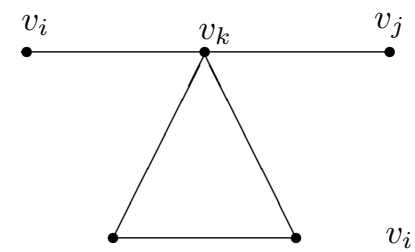

(a)

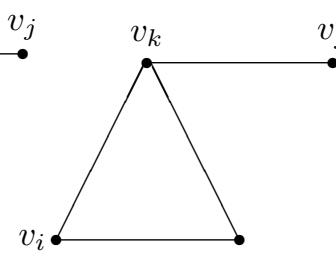

(b)

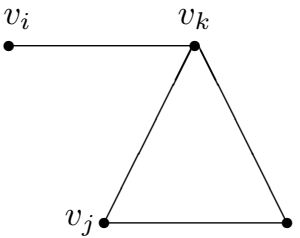

(c)

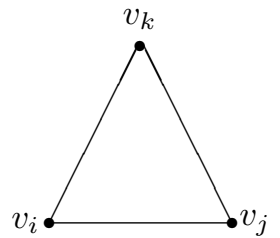

(d)

Fig 58

Case 20: For the configuration of Fig $59, \mathrm{~N}=6, \mathrm{M}=\left(\begin{array}{c}a_{i j}^{(2)} \\ 2\end{array}\right) a_{i j}$ and $\mathrm{F}=6\left(\begin{array}{c}a_{i j}^{(2)} \\ 2\end{array}\right) a_{i j}$.

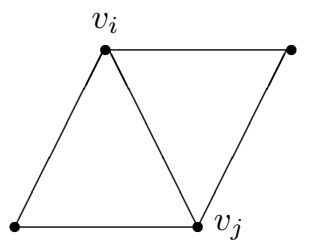

Fig 59 
Case 21: For the configuration of Fig $60, \mathrm{~N}=3, \mathrm{M}=\sum_{k=1, k \neq i, j}^{n}\left(a_{i k}^{(2)}-a_{i j}\right) a_{j k} a_{i j}$ and $\mathrm{F}=3 \sum_{k=1, k \neq i, j}^{n}\left(a_{i k}^{(2)}-a_{i j}\right) a_{j k} a_{i j}$. (See Theorem 1.10)

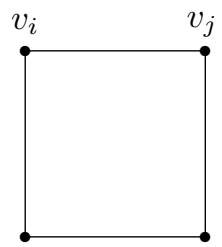

Fig 60

Case 22: For the configuration of Fig $61(\mathrm{a}), \mathrm{N}=2, \mathrm{M}=\frac{1}{2}\left(a_{i i}^{(4)}-a_{i i}^{(2)}-2\left(\begin{array}{c}d_{i} \\ 2\end{array}\right)-\sum_{k=1, k \neq i}^{n} a_{i k}^{(2)}\right) a_{i j}$ (See Theorem 2.1). Let $P_{1}$ denote the number of subgraphs of $G$ that have the same configuration as the graph of Fig $61(b)$ and are counted in M. Thus $\mathrm{P}_{1}=1 \times\left(\begin{array}{c}a_{i j}^{2} \\ 2\end{array}\right) a_{i j}$, where $\left(\begin{array}{c}a_{i j}^{2} \\ 2\end{array}\right) a_{i j}$ is the number of subgraphs of $\mathrm{G}$ that have the same configuration as the graph of Fig $61(\mathrm{~b})$ and this subgraph is counted only once in $\mathrm{M}$. Let $\mathrm{P}_{2}$ denote the number of subgraphs of $\mathrm{G}$ that have the same configuration as the graph of Fig 61(c) and are counted in M. Thus $\mathrm{P}_{2}=1 \times \sum_{k=1, k \neq i, j}^{n}\left(a_{i k}^{(2)}-a_{i j}\right) a_{j k} a_{i j}$, where $\sum_{k=1, k \neq i, j}^{n}\left(a_{i k}^{(2)}-a_{i j}\right) a_{j k} a_{i j}$ is the number of subgraphs of $\mathrm{G}$ that have the same configuration as the graph of Fig 61(c) (See Theorem 1.10) and this subgraph is counted only once in M. Consequently, $\mathrm{F}=\left(a_{i i}^{(4)}-a_{i i}^{(2)}-2\left(\begin{array}{c}d_{i} \\ 2\end{array}\right)-\sum_{k=1, k \neq i}^{n} a_{i k}^{(2)}\right) a_{i j}-2\left(\begin{array}{c}a_{i j}^{2} \\ 2\end{array}\right) a_{i j}-2 \sum_{k=1, k \neq i, j}^{n}\left(a_{i k}^{(2)}-a_{i j}\right) a_{j k} a_{i j}$.

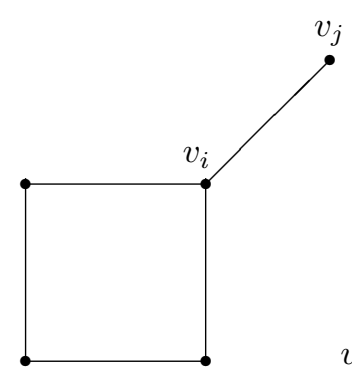

(a)

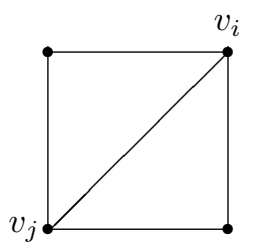

(b)

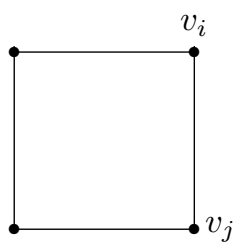

(c)

Fig 61

Case 23: For the configuration of Fig $62(\mathrm{a}), \mathrm{N}=2, \mathrm{M}=\frac{1}{2}\left(a_{j j}^{(4)}-a_{j j}^{(2)}-2\left(\begin{array}{c}d_{j} \\ 2\end{array}\right)-\sum_{k=1, k \neq j}^{n} a_{j k}^{(2)}\right) a_{i j}$ (See Theorem 2.1). Let $\mathrm{P}_{1}$ denote the number of subgraphs of $\mathrm{G}$ that have the same configuration as the graph of Fig $62(\mathrm{~b})$ and are counted in $\mathrm{M}$. Thus $\mathrm{P}_{1}=1 \times\left(\begin{array}{c}a_{i j}^{(2)} \\ 2\end{array}\right) a_{i j}$, where $\left(\begin{array}{c}a_{i j}^{(2)} \\ 2\end{array}\right) a_{i j}$ is the number of subgraphs of $\mathrm{G}$ that have the same configuration as the graph of Fig $62(\mathrm{~b})$ and this subgraph is counted only once in $\mathrm{M}$. Let $\mathrm{P}_{2}$ denote the number of subgraphs of $\mathrm{G}$ that have the same configuration as the graph of Fig 62(c) and are counted in M. Thus $\mathrm{P}_{2}=1 \times \sum_{k=1, k \neq i, j}^{n}\left(a_{i k}^{(2)}-a_{i j}\right) a_{j k} a_{i j}$, where $\sum_{k=1, k \neq i, j}^{n}\left(a_{i k}^{(2)}-a_{i j}\right) a_{j k} a_{i j}$ is the number of subgraphs of $\mathrm{G}$ that have the same configuration as the graph of Fig 62(c) (See Theorem 1.10) and this subgraph is counted only once in M. Consequently, $\mathrm{F}=\left(a_{j j}^{(4)}-a_{j j}^{(2)}-2\left(\begin{array}{c}d_{j} \\ 2\end{array}\right)-\sum_{k=1, k \neq j}^{n} a_{j k}^{(2)}\right) a_{i j}-2\left(\begin{array}{c}a_{i j}^{(2)} \\ 2\end{array}\right) a_{i j}-2 \sum_{k=1, k \neq i, j}^{n}\left(a_{i k}^{(2)}-a_{i j}\right) a_{j k} a_{i j}$. 


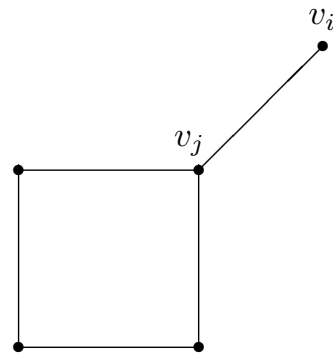

(a)

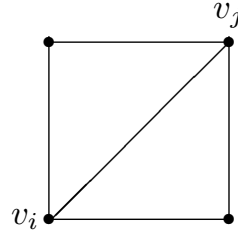

(b)

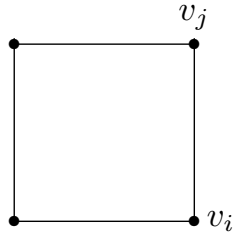

(c)

Fig 62

Now we add the values of $\mathrm{F}$ arising from the above cases and determine $x$. Substituting the value of $x$ in $a_{i j}^{(5)}-x$ and simplifying, we get the desired result.

Example 2.8 In the graph of Fig 39, $a_{12}^{(5)}=521,\left(2 d_{1}+2 d_{2}+d_{1} d_{2}+a_{11}^{(4)}+a_{22}^{(4)}-a_{11}^{(2)}-a_{22}^{(2)}-a_{12}^{(2)}-2\left(\begin{array}{c}d_{1} \\ 2\end{array}\right)-2\left(\begin{array}{c}d_{2} \\ 2\end{array}\right)\right.$

$\left.+2\left(\begin{array}{c}d_{1}-1 \\ 2\end{array}\right)+2\left(\begin{array}{c}d_{2}-1 \\ 2\end{array}\right)-6\left(\begin{array}{c}a_{12}^{(2)} \\ 2\end{array}\right)-4\right) a_{12}=185,\left(a_{11}^{(3)}+a_{22}^{(3)}\right) a_{12}^{(2)}=160, \sum_{k=2}^{6} a_{1 k}^{(2)} a_{12}=20$,

$\sum_{k=1, k \neq 2}^{6} a_{2 k}^{(2)} a_{12}=20, \sum_{k=3}^{6} a_{k k}^{(3)} a_{1 k} a_{2 k}=80, \sum_{k=3}^{6}\left(a_{1 k}^{(2)}+a_{2 k}^{(2)}-a_{1 k}-a_{2 k}-2 a_{1 k} a_{2 k}\right) a_{12}=16$,

$\sum_{k=3}^{6}\left(a_{1 k}^{(2)}-a_{12}\right)\left(3 a_{12}+3 a_{1 k}-d_{1}-d_{2}-d_{k}+1\right) a_{2 k}=-96, \sum_{k=3}^{6}\left(a_{2 k}^{(2)}-a_{12}\right)\left(3 a_{2 k}-d_{k}+2\right) a_{1 k}=0$.

So, by Theorem 2.7, the number of $v_{1}-v_{2}$ paths of length 5 in the graph of Fig 39 is 24.

\section{References}

[1] Nazanin Movarraei and M. M. Shikare, On the number of paths of lengths 3 and 4 in a graph, International Journal of Applied Mathematical Research, 3(2) (2014), 178-189.

[2] F. Harary and B. Manvel, On the number of cycles in a graph, Mat. Casopis Sloven. Akad. Vied 21 (1971),55-63.

[3] N. Alon, R. Yuster and U. Zwick, Finding and counting given length cycles, Algorithmica, 17(1997), 209-223.

[4] I.Tomescu, On the number of paths and cycles for almost all graphs and digraphs, Combinatorica, 6(1) (1986), 73-79.

[5] Eric T. Bax, Algorithms to count paths and cycles, Inf. Process. Lett, 52 (1994), 249-252.

[6] Noga Alon, Raphy Yuster and Uri Zwick, Color-coding: a new method for finding simple paths, cycles and other small subgraphs within large graphs, ACM Newyork, 1994, 326-335.

[7] Eric Bax and Joel Franklin, A finite-difference sieve to count paths and cycles by length, Inf. Process. Lett, 60(4) (1996), 171-176.

[8] Ben Roberts and Drik P. Kroese, Estimating the number of s-t paths in a graph, J. Graph Algorithms and Applications, 11(1) (2007), 195-214.

[9] Ryan Williams, Finding a path of length $\mathrm{k}$ in $O^{*}\left(2^{K}\right)$ time, Inf. Process. Lett, 109(6) (2009), 315-318.

[10] Andreas Bjorklund, Thore Husfeldt, Petteri Kaski and Mikko Koivisto, Counting paths and packing in halves, Lecture Notes in Computer Science, 5757 (2009), 578-586.

[11] Eric. T. Bax, Inclusion and exclusion algorithm for the Hamiltonian path problem, Inform. Process. Lett. 27(4) (1993), 203-207.

[12] A. Bjorklund, T. Husfeldt, P. Kaski, M. Koivisto, The fast intersection transform with applications to counting paths, CoRR, abs/0809.2489 (2008). 
[13] J. Chen, S. Lu, S. H. Sze, F. Zhang, Improved algorithms for path, matching and packing problems, 18th Annual ACMSIAM Symposium on Discrete Algorithms (SODA 2007), Philadelphia, PA, USA, PP. 298-307. Society for Industrial and Applied Mathematics (2007).

[14] Y. Gurevich, S. Shelah, Expected computation time for Hamiltonian path problem, SIAM J. Comput, 16(1987), 486-502.

[15] I. Koutis, Faster algebraic algorithm for path and packing problems, CALP (2008), LNCS 5125, 575-586, Springer.

[16] D. J. A. Welsh, Complexity: Knots, Colouring and counting, Cambridge University Press, 1993.

[17] Eric. T. Bax, Improvements and bounding for the inclusion and exclusion Hamiltonian algorithm, Caltech-CS-TR-94-11.

[18] B. Monien, How to find long paths efficiently, Annals of Discrete Mathematics, volume 25, (1985), 239-254.

[19] D. Richards, Finding short cycles in a planar graph using separators, Journal of Algorithms, volume 7, (1974), 382-394.

[20] J.A. Bondy and M. Simonovit, Cycles of even length in graphs, Journal of Combinatorial Theory Series B, volume 16, (1974), 97-105.

[21] Lowell W. Beineke and Robin J. Wilson, Topics in Algebraic Graph Theory, Cambridge University Press, 2007.

[22] F. Harary, Graph Theory, Addison-Wesley, Reading, Mass, 1969.

[23] G. Baroti, On the number of certain hamilton circuits of a complete graph, Periodica Math. Hung, 3 (1973), $135-139$. 